\title{
Amorphous In-Ga-Zn-O thin-film transistor active pixel sensor $x$-ray imager for digital breast tomosynthesis
}

\author{
Chumin Zhao and Jerzy Kanickia) \\ Solid-State Electronic Laboratory, Department of Electrical Engineering and Computer Science, \\ University of Michigan, Ann Arbor, Michigan 48109
}

(Received 27 November 2013; revised 21 May 2014; accepted for publication 23 July 2014; published 12 August 2014)

\begin{abstract}
Purpose: The breast cancer detection rate for digital breast tomosynthesis (DBT) is limited by the $\mathrm{x}$ ray image quality. The limiting Nyquist frequency for current DBT systems is around $5 \mathrm{lp} / \mathrm{mm}$, while the fine image details contained in the high spatial frequency region $(>5 \mathrm{lp} / \mathrm{mm})$ are lost. Also today the tomosynthesis patient dose is high $(0.67-3.52 \mathrm{mGy})$. To address current issues, in this paper, for the first time, a high-resolution low-dose organic photodetector/amorphous In-Ga-Zn-O thin-film transistor (a-IGZO TFT) active pixel sensor (APS) x-ray imager is proposed for next generation DBT systems.
\end{abstract}

Methods: The indirect x-ray detector is based on a combination of a novel low-cost organic photodiode (OPD) and a cesium iodide-based (CsI:Tl) scintillator. The proposed APS x-ray imager overcomes the difficulty of weak signal detection, when small pixel size and low exposure conditions are used, by an on-pixel signal amplification with a significant charge gain. The electrical performance of a-IGZO TFT APS pixel circuit is investigated by SPICE simulation using modified Rensselaer Polytechnic Institute amorphous silicon (a-Si:H) TFT model. Finally, the noise, detective quantum efficiency (DQE), and resolvability of the complete system are modeled using the cascaded system formalism.

Results: The result demonstrates that a large charge gain of 31-122 is achieved for the proposed high-mobility $\left(5-20 \mathrm{~cm}^{2} / \mathrm{V} \mathrm{s}\right)$ amorphous metal-oxide TFT APS. The charge gain is sufficient to eliminate the TFT thermal noise, flicker noise as well as the external readout circuit noise. Moreover, the low TFT $\left(<10^{-13} \mathrm{~A}\right)$ and OPD $\left(<10^{-8} \mathrm{~A} / \mathrm{cm}^{2}\right)$ leakage currents can further reduce the APS noise. Cascaded system analysis shows that the proposed APS imager with a $75 \mu \mathrm{m}$ pixel pitch can effectively resolve the Nyquist frequency of $6.67 \mathrm{lp} / \mathrm{mm}$, which can be further improved to $\sim 10 \mathrm{lp} / \mathrm{mm}$ if the pixel pitch is reduced to $50 \mu \mathrm{m}$. Moreover, the detector entrance exposure per projection can be reduced from 1 to $0.3 \mathrm{mR}$ without a significant reduction of DQE. The signal-to-noise ratio of the a-IGZO APS imager under $0.3 \mathrm{mR}$ x-ray exposure is comparable to that of a-Si:H passive pixel sensor imager under $1 \mathrm{mR}$, indicating good image quality under low dose. A threefold reduction of current tomosynthesis dose is expected if proposed technology is combined with an advanced DBT image reconstruction method.

Conclusions: The proposed a-IGZO APS x-ray imager with a pixel pitch $<75 \mu \mathrm{m}$ is capable to achieve a high spatial frequency $(>6.67 \mathrm{lp} / \mathrm{mm})$ and a low dose $(<0.4 \mathrm{mGy})$ in next generation DBT systems. (c) 2014 American Association of Physicists in Medicine. [http://dx.doi.org/10.1118/1.4892382]

Key words: digital breast tomosynthesis, amorphous In-Ga-Zn-O thin-film transistor, active pixel sensor, cascaded system analysis, detective quantum efficiency

\section{INTRODUCTION}

Standard two-dimensional (2D) mammography x-ray breast imaging technology suffers a limited detection rate due to severe breast tissue overlap. ${ }^{1}$ Digital breast tomosynthesis (DBT), as an alternative to mammography, eliminates the breast tissue superposition issue of mammography by reconstructing a series of tomographic cross-section images. ${ }^{2-4}$ Current DBT systems consist of a moveable x-ray source, a photodetector, and an active-matrix thin-film transistor (TFT) array. ${ }^{5-7}$ The $\mathrm{x}$-ray source motion for tomosynthesis can be either "step and shoot" or "continuous." 8 The step and shoot motion reduces image blur due to x-ray source motion, while increases image blur due to human motion with increased total scan time. ${ }^{8,9}$ During the acquisition time (3.7-25 s), 9-25 low-dose projection images are obtained from a total scan angle from $15^{\circ}$ to $45^{\circ} .{ }^{9,10}$ Increasing the total scan angle provides better vertical resolution. However, large number of projection images will prolong the total scan time and will increase the total dose. ${ }^{9}$ Optimizing the image acquisition parameters for low dose is still under research. ${ }^{8,11,12}$

The technical specifications of several clinically approved DBT systems (GE Essential, Hologic Selenia Dimensions, and Siemens Mammomat Inspiration) and the proposed next generation tomosynthesis system are shown in Table I. ${ }^{5-7,9,10}$

Clinically, the obtained image quality needs to be optimized for radiologists to distinguish microcalcification details. ${ }^{13}$ The limiting spatial frequency (Nyquist frequency) is defined as $1 /\left(2 a_{\text {pix }}\right)$, where $a_{\text {pix }}$ is the pixel pitch. A pixel pitch of $\sim 100 \mu \mathrm{m}$ corresponds to the Nyquist frequency 
TABLE I. Technical specifications of digital breast tomosynthesis imaging systems.

\begin{tabular}{|c|c|c|c|c|}
\hline & GE Essential $^{\mathrm{a}}$ & $\begin{array}{c}\text { Hologic Selenia } \\
\text { Dimensions }\end{array}$ & $\begin{array}{l}\text { Siemens Mammomat } \\
\text { Inspiration }\end{array}$ & Proposed system \\
\hline Detector & $\begin{array}{l}\text { CsI:Tl/a-Si:H p-i-n } \\
\text { (indirect) }\end{array}$ & a-Se (direct) & a-Se (direct) & $\begin{array}{l}\text { CsI:Tl/organic photodiode } \\
\text { (indirect) }\end{array}$ \\
\hline TFT technology & a-Si:H TFT & a-Si:H TFT & a-Si:H TFT & a-InGaZnO TFT \\
\hline Detector size $(\mathrm{cm})$ & $24 \times 30$ & $24 \times 29$ & $24 \times 30$ & $24 \times 30$ \\
\hline Pixel resolution & $2394 \times 3062$ & $1664 \times 2048(2 \times 2$ binning $)$ & $2816 \times 3584$ & $3200 \times 4000$ \\
\hline Pixel layout & Side by side & Side by side & Side by side & Vertically stacked \\
\hline Pixel pitch $(\mu \mathrm{m})$ & 100 & $140(2 \times 2$ binning $)$ & 85 & $<75$ \\
\hline Pixel fill factor & $0.5-0.8$ & $0.5-0.8$ & $0.5-0.8$ & $>0.8$ \\
\hline Limiting spatial resolution (lp/mm) & $\sim 5$ & $\sim 5$ & $\sim 6$ & $>6.67$ \\
\hline Pixel circuit & Passive pixel sensor & Passive pixel sensor & Passive pixel sensor & Active pixel sensor \\
\hline Scan time (s) & 7 & 3.7 & 25 & $<4$ \\
\hline Total scan angle & $25^{\circ}$ & $15^{\circ}$ & $45^{\circ}$ & NA \\
\hline X-ray source motion & Step and shoot & Continuous & Continuous & Continuous \\
\hline Detector motion & Static & Rotating & Static & Rotating \\
\hline Number of views & 9 & 15 & 25 & NA \\
\hline Mean glandular dose (mGy) & NA & $0.67-2.64^{\mathrm{b}}$ & NA & $<0.4^{\mathrm{c}}$ \\
\hline
\end{tabular}

${ }^{a}$ The next generation imager is GE SenoClaire. No technical details are available.

${ }^{\mathrm{b}}$ The MGD is for breasts of $2-8 \mathrm{~cm}$ thickness and $50 \%$ glandular fraction. The dose is $1.3 \mathrm{mGy}$ for an average breast (50\% glandular fraction, $5 \mathrm{~cm}$ thick) and can be as high as $3.52 \mathrm{mGy}$ for dense breasts.

${ }^{c}$ Cascaded system analysis shows that the detector x-ray exposure can be reduced by threefold without influencing the image quality significantly using the APS imager. Therefore, a $3 \times$ dose reduction is expected in combination with an advanced image reconstruction method.

( $f_{\text {Nyquist }}$ ) of $5 \mathrm{lp} / \mathrm{mm}$ due to the aliasing. ${ }^{14}$ Higher spatial frequency $(>5 \mathrm{lp} / \mathrm{mm})$ can provide fine image details that should not be lost. A pixel pitch of $<75 \mu \mathrm{m}$ is required to resolve spatial frequency of $>6.67 \mathrm{lp} / \mathrm{mm}$. As shown in Table I, DBT systems with pixel pitch around $100 \mu \mathrm{m}$ have been developed based on amorphous silicon (a-Si:H) TFT passive pixel sensor (PPS). ${ }^{9,10,15,16}$ Hologic Selenia Dimensions detector has a pixel pitch of $70 \mu \mathrm{m}$ for mammography, but a $2 \times 2$ binning technology is generally used for DBT resulting in an effective pixel pitch of $140 \mu \mathrm{m} .{ }^{10}$ In PPS, it is difficult to further reduce the pixel size due to the weak signal caused by the low detector entrance exposure per projection $(\sim 1 \mathrm{mR})$ (Ref. 7) during tomosynthesis. We use the unit Roentgen $(\mathrm{R})$ to define the $\mathrm{x}$-ray exposure to the detector and Gray (Gy) to measure the patient glandular dose; $1 \mathrm{R}$ is equal to $8.76 \mathrm{mGy}$ of absorbed dose in dry air. For small pixel size and under low x-ray exposure conditions, the electronic noise becomes significant and will result in a limited signal-to-noise ratio (SNR) and a low detective quantum efficiency (DQE). ${ }^{17}$ In addition, the detectable input signal is further attenuated by the pixel fill factor $(F F)(0.4-0.8),{ }^{15,18}$ which is defined by the ratio of photodiode active area over the entire pixel area. As a result, the detector entrance exposure per projection $(\sim 1 \mathrm{mR})$ and patient mean glandular dose (MGD) remain high to compensate for weak signal. Feng et al. reported that the MGD for breasts of 2-8 cm thickness and 50\% glandular fraction is $0.67-2.64 \mathrm{mGy}(1.3 \mathrm{mGy}$ for a $5 \mathrm{~cm}$ thick average breast) and can be as high as $3.52 \mathrm{mGy}$ for dense breasts. ${ }^{6}$ The dose of a single-view DBT exam is about one to two times that of a single-view full-field digital mammogram (FFDM). ${ }^{19}$ The question of dose optimization for DBT has not been solved yet.
To achieve a high spatial frequency $(>6.67 \mathrm{lp} / \mathrm{mm})$ and a low patient dose $(<0.4 \mathrm{mGy})$, we propose a vertically integrated indirect organic photodetector/amorphous In-Ga$\mathrm{Zn}-\mathrm{O}$ (a-IGZO) TFT active pixel sensor (APS) x-ray imager for next generation low dose DBT systems. In contrast to PPS, APS amplifies the input signal on the pixel level and minimizes the influence of electronic noise by a charge gain..$^{20-22,25}$ The a-IGZO TFT array is vertically stacked in combination with a low-cost poly(3-hexylthiophene):phenyl$\mathrm{C}_{61}$-butyric acid methyl ester (P3HT:PCBM) organic photodiode (OPD) (Refs. 23-26) and a thallium-doped cesium iodide (CsI:Tl) scintillator, ${ }^{27,28}$ which addresses the diminishing $F F$ issue when small pixel size is used. The electrical performance of the proposed a-IGZO TFT APS is studied by SPICE circuit simulation using the Rensselaer Polytechnic Institute (RPI) a-Si:H TFT model modified for a-IGZO $\mathrm{TFT}^{29}$ The noise and DQE of the active pixel a-IGZO TFTbased high-resolution $\mathrm{x}$-ray imager are investigated using the cascaded system model in Sec. $4 .^{18,30-32}$

\section{X-RAY DETECTOR}

\section{A. Direct conversion detector}

In general, DBT systems can be categorized into direct (Hologic Selenia Dimensions, and Siemens Mammomat Inspiration) and indirect conversion detectors (GE Essential). ${ }^{9,10}$ For direct conversion detectors, as shown in Fig. 1(a), x-ray photons are absorbed by semiconductors such as amorphous selenium (a-Se) photodiodes and directly converted to electric signal. ${ }^{33,34}$ Compared to indirect detectors, direct conversion detectors eliminate problems associated with the light scattering of the scintillator, which can 
(a)

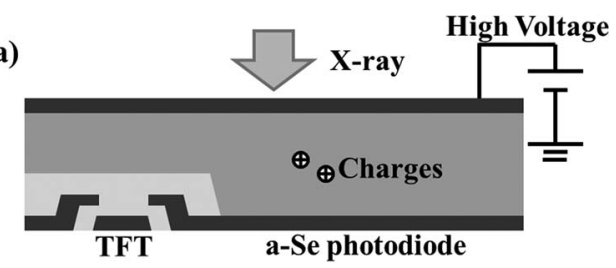

(b)

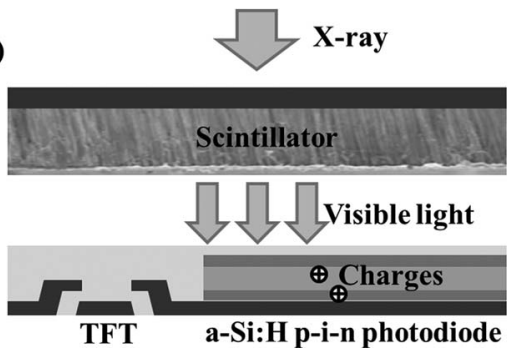

FIG. 1. Schematics of (a) direct and (b) indirect conversion detectors are shown. An a-Se photodiode and a conventional a-Si:H p-i-n photodiode are used for indirect and direct conversion detectors, respectively.

be an issue for high resolution DBT. ${ }^{33}$ The incident noise power spectrum (NPS) for direct conversion detectors extend well above the $f_{\text {Nyquist }}$. The aliasing and back-folding of NPS from spatial frequency above the $f_{\text {Nyquist }}$ degrades the SNR and DQE for spatial frequency region from zero to $f_{\text {Nyquist }} \cdot{ }^{14,35}$ The operational voltage for direct detectors is extremely high (typically $2 \mathrm{kV}$ for a $200 \mu \mathrm{m}$ a-Se photodiode), which can affect TFT and imager lifetime. ${ }^{33}$ In general, it takes longer time to read out the signal from a-Se detectors which are operated at high voltage. To reduce the total scan time and compensate the small exposure area per pixel, techniques such as $2 \times 2$ pixel binning was introduced. ${ }^{6,9,10}$ The pixel binning will increase the overall signal and reduce the scan time, but at the same time will reduce imager resolution. Zhao et al. indicated that the doubled pixel pitch $(70 \mu \mathrm{m} \times 2=140 \mu \mathrm{m}$ for Hologic Selenia Dimensions detector $)^{6}$ affects the image resolution. $^{7}$ The modulation transfer function (MTF) of the $140 \mu \mathrm{m}$ binned direct Hologic Selenia Dimensions detector is comparable to that of a $100 \mu \mathrm{m}$ indirect CsI:Tl/a-Si:H PPS detector. ${ }^{48}$ For indirect DBT detectors, electronic noise considerations limit the readout rate at a $2-3 \times$ faster rate than for a-Se. Therefore, indirect DBT detectors do not require pixel binning and can achieve higher spatial resolution and faster scan time.

\section{B. Indirect conversion detector}

Indirect detectors have the greatest potential to achieve improved cancer detection performance and will allow patient dose below that of conventional mammography. For indirect conversion detectors, as shown in Fig. 1(b), first absorbed $x$ rays by a scintillating material generate visible light, that is detected next by a photodiode. ${ }^{36,37}$ Indirect detection systems commonly consist of a scintillator such as CsI:Tl or terbiumdoped gadolinium oxysulfide $\left(\mathrm{Gd}_{2} \mathrm{O}_{2} \mathrm{~S}: \mathrm{Tb}\right)$ and an amorphous silicon (a-Si:H) p-i-n photodiode. ${ }^{5,15-18}$ The scintillators have a strong $\mathrm{X}$-ray absorption properties generating $>4 \times 10^{4}$ photons/ $\mathrm{MeV}$ and a fast response time in the range of microsecond to millisecond. ${ }^{38,39}$ Therefore, indirect conversion detectors are suitable to be operated at a low dose condition and a short scan time $(<4 \mathrm{~s})$. Although in such detector it is expected that the MTF will reduce the signal due to scintillator scattering, but at the same time, the quantum noise will also be affected and the SNR will not be significantly influenced. ${ }^{35}$ Currently, the fabrication cost for both a-Si:H pi-n or a-Se photodetectors can be high. A cost-effective flexible or deformable x-ray imager is very desirable for next generation DBT.

As an alternative to conventional a-Si:H p-i-n or a-Se photodiodes, OPD appears to be an potential candidate for next generation $x$-ray imagers due to the low-cost large-area fabrication process. ${ }^{40,41}$ In addition, the thin OPDs $(<1 \mu \mathrm{m})$ can be fabricated on flexible/deformable substrates that could lead to next generation flexible/deformable x-ray imagers. ${ }^{42}$ Bulk heterojunction (BJT) OPDs based on P3HT:PCBM are being intensely investigated for $\mathrm{x}$-ray detectors. ${ }^{23-26} \mathrm{~A}$ low dark current of $<10^{-8} \mathrm{~A} / \mathrm{cm}^{2}$, a high external quantum efficiency (EQE) of $>60 \%$, a rise/fall time in the millisecond range, an $\mathrm{x}$-ray robustness of $15 \mathrm{~Gy}$ accumulated dose, and a suitable lifetime have been achieved, which are acceptable for tomosynthesis. ${ }^{23,24,26}$ The current-voltage (I-V) characteristics of the P3HT:PCBM OPDs in dark ambient and under illumination as well as the EQE are shown in Fig. 2(a), the data reported by Arca et al. from Siemens AG are also shown. ${ }^{23}$ Also the absorption spectrum of P3HT:PCBM (Ref. 43) fits well the emission spectrum of both CsI:Tl (Ref. 44) and $\mathrm{Gd}_{2} \mathrm{O}_{2} \mathrm{~S}: \mathrm{Tb}$ (Ref. 45) scintillators [Fig. 2(b)], which demonstrates its viability for indirect $\mathrm{x}$-ray detection in combination with either of these two scintillators. Among various scintillators, CsI:Tl shows a high light output $(\sim 6 \times$ $10^{4}$ photons $\left./ \mathrm{MeV}\right)$, a fast response time $(\sim 1 \mu \mathrm{s})$, a low refractive index (1.79), and a proper emission spectra $\left(\lambda_{\text {em }}\right.$ $\sim 550 \mathrm{~nm}),{ }^{38,39}$ which appears to be an optimal choice as the scintillator in combination with the OPD. Besides, the columnar structure of CsI:Tl reduces lateral light scattering within the scintillator and can provide better MTF for a high resolution imager.

In this work, indirect $\mathrm{x}$-ray detector using the P3HT:PCBM top-cathode OPD combined with the CsI:Tl scintillator is proposed. A commercial $150 \mu \mathrm{m}$ structured high light output (HL) CsI:Tl scintillator deposited on a fiber optic faceplate (FOP), referred as a fiber optic scintillator (FOS), can be integrated on top of the imaging device. ${ }^{46,47}$ The scintillator can also be deposited directly over OPD as long as the deposition temperature is below $150^{\circ} \mathrm{C}$. An OPD using a $100 \mathrm{~nm}$ sputtered Au layer as the bottom anode and a very thin thermal evaporated $\mathrm{Ca} / \mathrm{Ag}(3 \mathrm{~nm} / 10 \mathrm{~nm})$ layer as a semitransparent top cathode was reported. ${ }^{25}$ Indium-tin-oxide (ITO) and a very thin LiF/Al layer can also be used as the anode and cathode, respectively. A P3HT:PCBM active layer $(\sim 200 \mathrm{~nm})$ was sandwiched in between two electrodes. ${ }^{25}$ The solution processed P3HT:PCBM layer has to be fabricated on top of the hole-transporting interlayer to avoid being dissolved by any other solvents. Therefore, the topcathode OPD structure is suitable for this application. The 

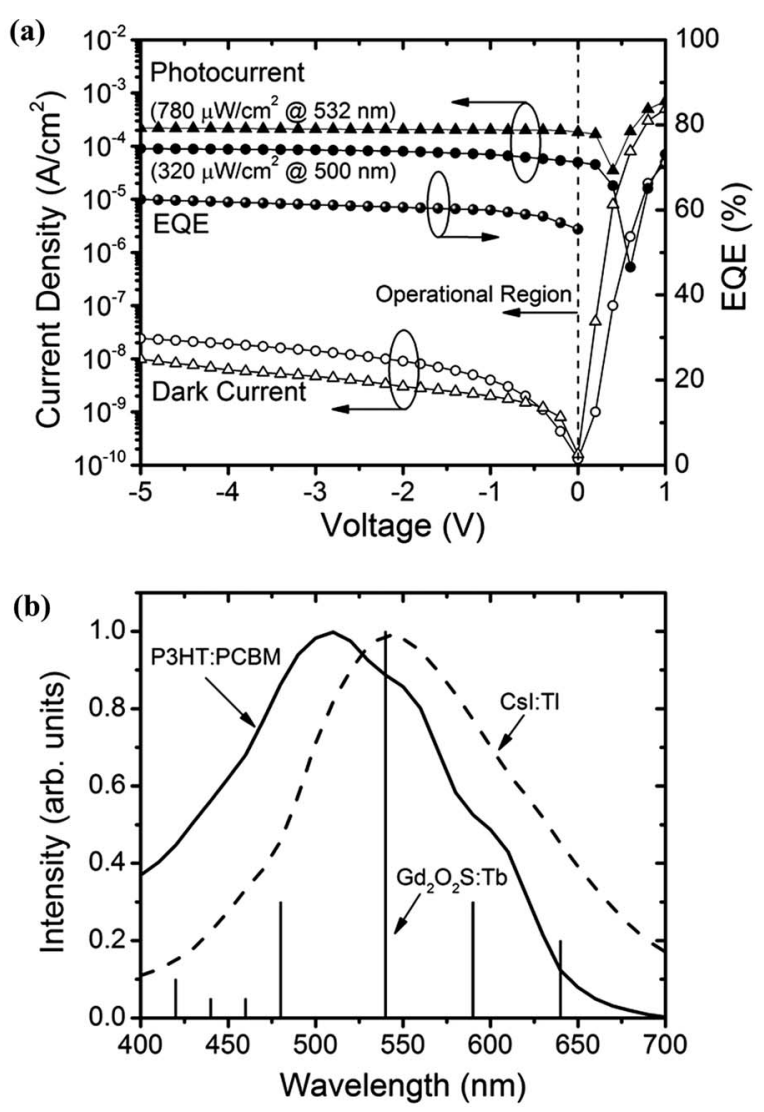

FIG. 2. (a) Current-voltage (I-V) characteristics of P3HT:PCBM organic photodiode in dark ambient and under light illumination (symbols: $\bigcirc, \mathbf{0})$ in comparison with the published dark current data by Arca et al. from Siemens AG (symbols: $\Delta, \mathbf{\Lambda}$ ) (Ref. 23) are shown. EQE under reverse bias condition is also shown. (b) P3HT:PCBM absorption spectrum (solid line) (Ref. 43), CsI:Tl (dashed line) (Ref. 44), and $\mathrm{Gd}_{2} \mathrm{O}_{2} \mathrm{~S}: \mathrm{Tb}$ (bars) (Ref. 45) emission spectrums are provided.

interlayer materials can be poly(3,4-ethylenedioxythiophene) poly(styrenesulfonate) (PEDOT:PSS), ${ }^{24}$ spray coated P3HT single layer, ${ }^{23}$ or triarylamine-based. ${ }^{25}$ The entire OPD structure and scintillator need to be encapsulated to prolong the lifetime. Instead of using a conventional PPS TFT backplane, an APS TFT array is proposed. The TFT array is covered by the large-area OPD using a vertically stacked architecture to achieve a pixel fill factor greater than 0.8 for a $75 \mu \mathrm{m}$ pixel pitch. The cross-sectional view of the proposed APS x-ray detection system is shown in Fig. 3. The pixel design and SPICE simulation of APS are discussed in Sec. 3.

\section{ACTIVE PIXEL THIN-FILM TRANSISTOR BACKPLANE}

\section{A. Active pixel sensor}

Current mammography/DBT systems are based on a single TFT passive pixel sensor. The PPS has demonstrated its success for mammography $\mathrm{x}$-ray imagers due to the small pixel size $(<100 \mu \mathrm{m})$ and high detector entrance exposure $(\sim 10 \mathrm{mR}))^{5,7,18}$ However, for low dose and high resolution DBT applications, detection of the weak input pixel signal per projection is challenging. To realize low dose DBT, we need to achieve the following: (i) a low x-ray skin entrance expo-

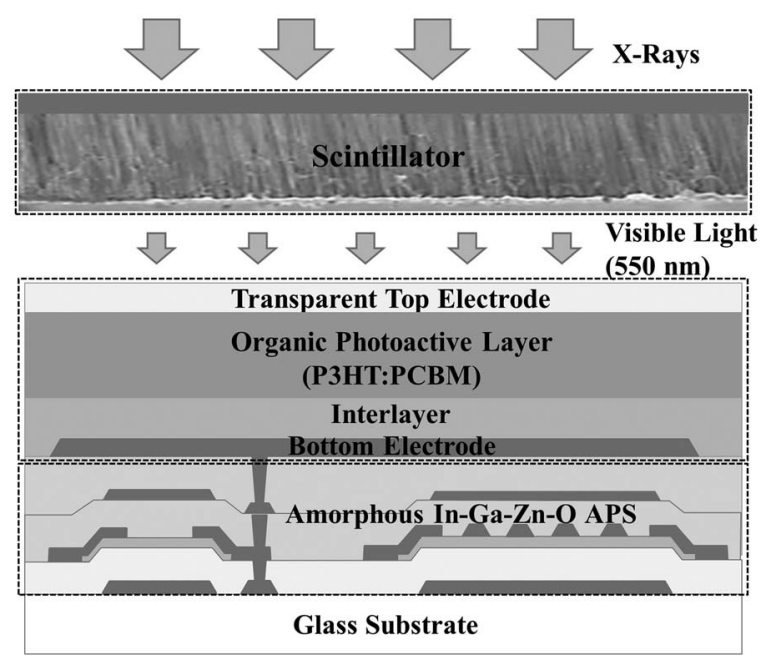

FIG. 3. Cross-sectional schematic of the proposed active pixel sensor x-ray imager based on amorphous In-Ga-Zn-O TFT backplane stacked by a large area P3HT:PCBM organic photodiode and CsI:Tl scintillator.

sure; (ii) a high $\mathrm{x}$-ray to photon to electron conversion efficiency (a high input electric signal); (iii) a significant detector output signal; and (iv) low quantum and electronic noise. The pixel pitch of DBT detectors is limited to around $100 \mu \mathrm{m} .{ }^{9,10}$ The bottleneck of reducing the pixel size and dose simultaneously can be solved by the proposed indirect OPD/a-IGZO APS imager as follow: (i) The reduced skin entrance exposure (low patient dose) results in a low detector entrance exposure; (ii) the combination of CsI:Tl with a high $\mathrm{x}$-ray to photon conversion efficiency $\left(\sim 6 \times 10^{4}\right.$ photons/MeV) and OPD with a high EQE $(>60 \%)$ ensures a high input signal under low dose; (iii) the input electric signal is amplified by the APS at the pixel level by a large charge gain $>30$ resulting in a significant output signal; (iv) the TFT thermal noise, flicker noise, and the dominant external readout circuit noise can be eliminated by the APS; the low off-current of the proposed a-IGZO TFT further reduces the pixel circuit noise, while the quantum noise is reduced by the low $\mathrm{x}$-ray exposure. The operation principle of APS is discussed below.

As an alternative pixel architecture, APS consists of three TFTs, namely, reset, readout, and amplifying TFT $\left(T_{\mathrm{RST}}, T_{\mathrm{READ}}\right.$, and $\left.T_{\mathrm{AMP}}\right)$, respectively. ${ }^{20,25}$ The APS circuit schematic with parasitic elements and external readout circuit is shown in Fig. 4(a). An APS pixel unit contains three TFTs and a photodiode with a capacitance $\left(C_{\mathrm{PD}}\right) . C_{\mathrm{PD}}$ serves as a storage capacitor in proposed pixel circuit. The output current of an individual pixel flows through the data line, which contains a parasitic resistance $\left(R_{\text {DATA }}\right)$ and capacitance $\left(C_{\text {DATA }}\right)$. Finally, the output signal is stored on the feedback capacitor $\left(C_{\mathrm{FB}}\right)$ of the switched-capacitor amplifier (Burr-Brown ACF2101) used as the external readout circuit.

APS operation consists of three distinct modes: reset, integration, and readout. ${ }^{20,25}$ In the reset mode, $T_{\text {RESET }}$ is $\mathrm{ON}$, while $T_{\mathrm{READ}}$ is OFF. The voltage on $V_{\mathrm{S}}$ node is set to $V_{\mathrm{REF}}$, which should be larger than the threshold voltage of $T_{\mathrm{AMP}}$. During the integration time, $t_{\text {int }}$, a short-pulse x-ray projection induces a photocurrent by the scintillator photodiode combination. The $\mathrm{x}$ rays are converted to photons in the CsI:Tl 
(a)

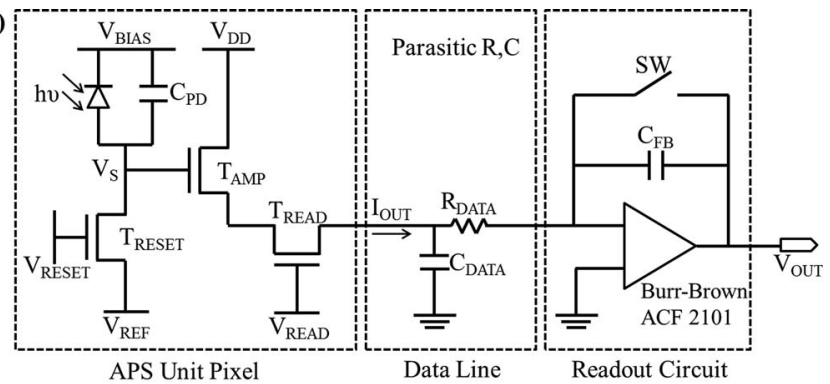

(b)

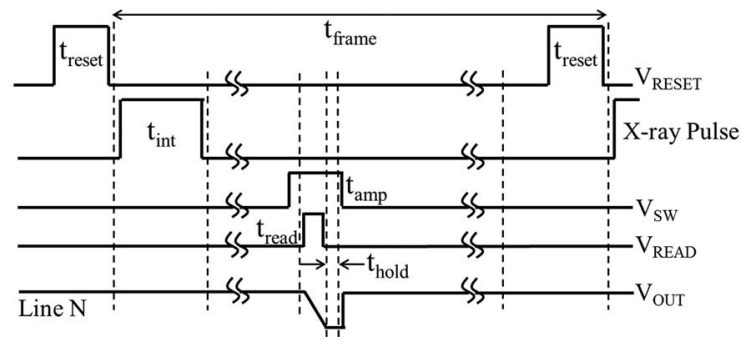

FIG. 4. (a) Top-cathode APS circuit schematic with parasitic elements and external readout circuit is shown. The APS unit pixel consists of three transistors ( $T_{\mathrm{RESET}}, T_{\mathrm{READ}}$, and $T_{\mathrm{AMP}}$ ) and a photodiode with a capacitance of $C_{\mathrm{PD}}$. (b) An example of the driving/reading scheme is also shown. Three modes, namely, reset $\left(t_{\text {reset }}\right)$, integration $\left(t_{\text {int }}\right)$, and readout $\left(t_{\text {read }}\right)$ are contained in a single frame. $t_{\mathrm{amp}}$ and $t_{\text {hold }}$ are the switched-capacitor amplifier ON time and readout signal hold time, respectively. $V_{\mathrm{BIAS}}, V_{\mathrm{DD}}$, and $V_{\mathrm{REF}}$ are constant voltage biases; $V_{\mathrm{SW}}$ is used to reset the charge on $C_{\mathrm{FB}}$ after output charge readout.

scintillator with a quantum efficiency of $6.6 \times 10^{4}$ photons $/ \mathrm{MeV}$. The photons propagate inside the columnar needles to the OPD with good image fidelity. The photons are converted into electrical signal (induced charge) inside the OPD with an EQE $>60 \%$. The induced charge is accumulated on $C_{\mathrm{PD}}$ and raises the voltage on the $V_{\mathrm{S}}$ node. In this stage, the quantum efficiency of the scintillator and photodiode, quantum noise (photon shot noise), OPD dark current shot noise, and TFT leakage current shot noise all contribute to the input SNR. After integration, $T_{\text {READ }}$ is turned $\mathrm{ON}$ and the output current $\left(I_{\text {OUT }}\right)$ is flowing through the data line, with parasitic $\mathrm{RC}$ components, into the external readout circuit. The stored charge on the $C_{\mathrm{FB}}$ is read line by line during the readout mode $\left(t_{\text {read }}\right)$ by external electronics. Figure 4(b) gives an example of the driving/reading scheme of an APS x-ray imager. The top view layout and vertical cross-section view of a $100 \mu \mathrm{m}$ forkshaped APS pixel is shown in Fig. 5 as an example. The forkshaped or half-Corbino TFTs can be used to maximize the channel width/length $(W / L)$ of TFTs to achieve a large charge gain within a limited pixel area. The $W / L$ of the $T_{\mathrm{RESET}}, T_{\mathrm{AMP}}$, and $T_{\mathrm{READ}}$ are $25 / 5,150 / 5$, and $150 \mu \mathrm{m} / 5 \mu \mathrm{m}$, respectively.

APS provides a charge gain to amplify the input signal on the pixel level to minimize the influence of electronic noise from both internal pixel circuit and external readout circuit. ${ }^{20-22,25}$ The electronic noise elements of APS can be categorized into preamplifying noise and postamplifying noise. ${ }^{20,49}$ The preamplifying electronic noise includes the photodiode dark current shot noise, $T_{\text {RESET }}$ leakage current shot noise, and reset noise. ${ }^{20,49}$ These noise elements cannot be reduced by APS. The postamplifying noise contains ther-

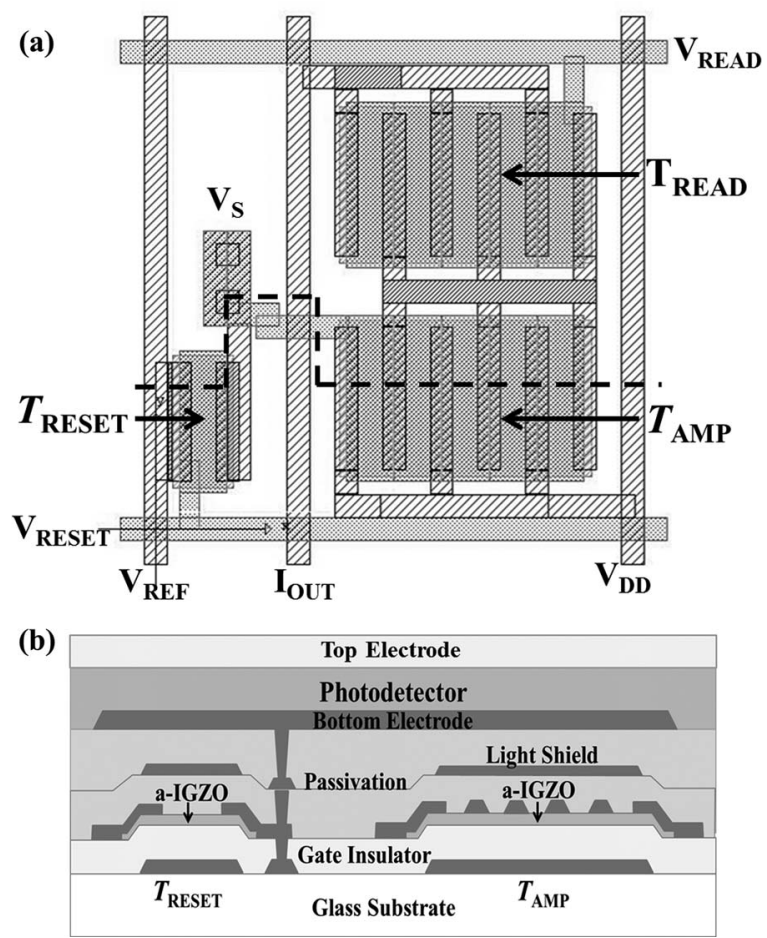

FIG. 5. Top view layout (a) and vertical cross-sectional view (b) of a $100 \mu \mathrm{m}$ fork-shaped APS pixel. The channel width/length $(W / L)$ of the reset TFT ( $\left.T_{\text {RESET }}\right)$, amplifying TFT $\left(T_{\mathrm{AMP}}\right)$, and readout TFT $\left(T_{\mathrm{READ}}\right)$ are $25 / 5,150 / 5$, $150 \mu \mathrm{m} / 5 \mu \mathrm{m}$, respectively.

mal and flicker noise of $T_{\mathrm{AMP}}$ and $T_{\mathrm{READ}}$, as well as the external readout circuit noise due to the operational amplifier..$^{20,49}$ The postamplifying noise elements can be eliminated by proposed a-IGZO APS and result in an improved output SNR but will not improve the input SNR. The charge gain is defined by the ratio of the output charge variations $\left(\Delta Q_{\text {out }}\right)$ on the feedback capacitor $\left(C_{\mathrm{FB}}\right)$ to the accumulated input charges $\left(\Delta Q_{\text {in }}\right)$ on the photodiode capacitor $\left(C_{\mathrm{PD}}\right)$. The totally generated number of charges from APS is related to the output current ( $\left.I_{\mathrm{OUT}}\right)$ of $T_{\mathrm{AMP}}$ and the readout time $\left(t_{\text {read }}\right)$. Suppose the dimensions $(W / L)$ of $T_{\mathrm{AMP}}$ and $T_{\mathrm{READ}}$ are the same and the output current is mainly determined by $T_{\mathrm{AMP}}$, the charge gain is given by ${ }^{20}$

$$
\begin{aligned}
G & =\left|\frac{\Delta Q_{\text {out }}}{\Delta Q_{\text {in }}}\right|=\left|\frac{\Delta I_{\mathrm{OUT}}}{\Delta V_{\mathrm{S}}}\right| \frac{t_{\text {read }}}{C_{\mathrm{PD}}}=g_{\mathrm{m}} \frac{t_{\text {read }}}{C_{\mathrm{PD}}} \\
& =\mu_{\text {eff }} \frac{W_{\mathrm{AMP}}}{L} \frac{C_{\mathrm{ox}}}{C_{\mathrm{PD}}} V_{\mathrm{DS} \_\mathrm{AMP}} t_{\text {read }},
\end{aligned}
$$

where $g_{\mathrm{m}}$ is the transconductance of $T_{\mathrm{AMP}}, \mu_{\mathrm{eff}}$ is the fieldeffect mobility TFTs, $W_{\mathrm{AMP}} / L$ is the channel width/length of $T_{\mathrm{AMP}}, C_{\mathrm{ox}}$ is the gate capacitance per unit area, $C_{\mathrm{PD}}$ is the capacitance of photodiode, $V_{\mathrm{DS} \_ \text {AMP }}$ is the drain to source voltage of $T_{\mathrm{AMP}}$, and $t_{\text {read }}$ is the readout time. For $V_{\mathrm{DS} \_\mathrm{AMP}}$ $>V_{\mathrm{GS} \_\mathrm{AMP}}-V_{\mathrm{T}}$, maximum gain can be achieved and Eq. (1) becomes

$$
G=\mu_{\text {eff }} \frac{W_{\mathrm{AMP}}}{L} \frac{C_{\mathrm{ox}}}{C_{\mathrm{PD}}}\left(V_{\mathrm{GS} \_\mathrm{AMP}}-V_{\mathrm{T}}\right) t_{\text {read }},
$$

where $V_{\mathrm{GS} \_ \text {AMP }}$ is the gate to source voltage of $T_{\mathrm{AMP}}$ and $V_{\mathrm{T}}$ is the threshold voltage of the $T_{\mathrm{AMP}}$. However, in this case, 
TABLE II. List of parameters, driving scheme, driving voltages, and TFT dimensions used in SPICE simulation.

\begin{tabular}{lc}
\hline \hline Parameter & Value \\
\hline Pixel pitch $\left(a_{\text {pix }}\right)$ & $75 \mu \mathrm{m}$ \\
Photodiode capacitance $\left(C_{\mathrm{PD}}\right)$ & $1 \mathrm{pF}$ \\
Feedback capacitance $\left(C_{\mathrm{FB}}\right)$ & $100 \mathrm{pF}$ \\
$V_{\mathrm{BIAS}}$ & $15 \mathrm{~V}$ \\
$V_{\mathrm{REF}}$ & $10 \mathrm{~V}$ \\
$V_{\mathrm{DD}}$ & $5 \mathrm{~V}$ \\
$V_{\mathrm{RESET}}(\mathrm{OFF} / \mathrm{ON})$ & $-3 \mathrm{~V} / 15 \mathrm{~V}$ \\
$V_{\mathrm{READ}}(\mathrm{OFF} / \mathrm{ON})$ & $-3 \mathrm{~V} / 15 \mathrm{~V}$ \\
$V_{\mathrm{T}}$ & $5 \mathrm{~V}$ \\
$T_{\mathrm{AMP}}(W / L)$ & $40 \mu \mathrm{m} / 5 \mu \mathrm{m}$ \\
$T_{\mathrm{READ}}(W / L)$ & $40 \mu \mathrm{m} / 5 \mu \mathrm{m}$ \\
$T_{\mathrm{RST}}(W / L)$ & $25 \mu \mathrm{m} / 5 \mu \mathrm{m}$ \\
Reset time $\left(t_{\text {reset }}\right)$ & $7 \mathrm{~ms}$ \\
Charge integration time $\left(t_{\text {int }}\right)$ & $10 \mathrm{~ms}$ \\
Integrator ON time $\left(t_{\text {amp }}\right)$ & $40 \mu \mathrm{s}$ \\
Readout time $\left(t_{\text {read }}\right)$ & $20 \mu \mathrm{s}$ \\
Hold time $\left(t_{\text {hold }}\right)$ & $10 \mu \mathrm{s}$ \\
Frame time $\left(t_{\text {frame }}\right)$ & $145 \mathrm{~ms}$ \\
\hline \hline
\end{tabular}

the gain is not a constant and the output to input signal is not linear, since $V_{\mathrm{S}}$ and $V_{\mathrm{GS} \_\mathrm{AMP}}$ are increasing during light integration. Therefore, $T_{\mathrm{AMP}}$ is preferred to work in the linear region $\left(V_{\mathrm{DS} \_\mathrm{AMP}}<V_{\mathrm{GS} \_\mathrm{AMP}}-V_{\mathrm{T}}\right)$ to maintain linearity between input and output signals. As shown in Table II, in the simulation, $V_{\mathrm{S}}$ is between $V_{\mathrm{REF}}\left(10 \mathrm{~V}\right.$ after reset) and $V_{\mathrm{BIAS}}$ ( $15 \mathrm{~V}$ for fully charged $C_{\mathrm{PD}}$ ), $V_{\mathrm{DD}}$ and $V_{\mathrm{T}}$ are $5 \mathrm{~V}$. For this specific operating condition, we have $V_{\mathrm{DD}}<V_{\mathrm{S}}-V_{\mathrm{T}}$, hence $V_{\mathrm{DS} \_\mathrm{AMP}}<V_{\mathrm{GS} \_\mathrm{AMP}}-V_{\mathrm{T}}$, which is linear region condition.

To achieve a large charge gain, two TFT parameters need to be optimized based on Eq. (1). First, the value of TFT channel width/length $\left(W_{\mathrm{AMP}} / L\right)$ should be large. For high resolution X-ray imagers (e.g., $3200 \times 4000$ with a pixel pitch of $75 \mu \mathrm{m})$, the dimension of TFT $\left(W_{\mathrm{AMP}} / L\right.$ of $T_{\mathrm{AMP}} \sim 40 \mu \mathrm{m} /$ $5 \mu \mathrm{m})$ is limited by the small pixel size. In this case, the forkshaped $^{50}$ (Fig. 5) or half-Corbino ${ }^{51}$ TFT structure is a possible approach to achieve large $W_{\mathrm{AMP}} / L$ within a small pixel area. Second, a high TFT field-effect mobility ( $\mu_{\text {eff }}$ ) must be used to achieve a large charge gain without enlarging the TFT dimensions and pixel size. Third, the readout time can be reduced using high mobility TFT technology combined with the indirect detector. Consequently, the reduced total scan time leads to reduced human motion and low dose if continuous $\mathrm{X}$-ray illumination is used.

To evaluate the feasibility of different TFT technologies and optimize the pixel circuit design for proposed APS xray imager at the early stage, SPICE simulation is a powerful tool. In Sec. 3.B, the electrical properties of the APS pixel and readout circuits will be investigated. The charge gain can be extracted from simulation results.

\section{B. Active pixel sensor pixel circuit simulation}

The SPICE circuit simulations were performed using SILVACO SmartSpice simulator. First, the a-Si:H TFT character- istics were model by RPI a-Si TFT model. ${ }^{29}$ Then the RPI a-Si TFT model was modified for a-IGZO TFTs. SPICE parameters of a-IGZO TFT were extracted by fitting the calculated curves to experimental measured TFT current-voltage (I-V) characteristics. ${ }^{52}$ We used the SPICE model of diode in combination with a current source and photodiode capacitance for the OPD. The SPICE model for the switched-capacitor amplifier (Burr-Brown ACF2101) provided by the supplier ${ }^{53}$ was also integrated in the circuit simulation. The electronic noise is not included in the simulation and will be discussed numerically in Sec. 3.C.

We first simulated the APS pixel circuit performance based on a-Si:H TFTs. The switched-capacitor amplifier is first turned ON and then the readout TFT ( $\left.T_{\text {READ }}\right)$ is ON for $20 \mu \mathrm{s} .{ }^{25}$ All the simulation parameters, TFT dimensions and driving scheme ${ }^{25}$ referring to Fig. 4(b) are listed in Table II. The pixel pitch $\left(a_{\text {pix }}\right)$ is $75 \mu \mathrm{m}$, which is used to achieve a high Nyquist frequency $(\sim 6.67 \mathrm{lp} / \mathrm{mm})$. Smaller pixel size (e.g., $50 \mu \mathrm{m}$ ) can restrict the TFT dimensions $(W / L)$, and charge gain. It also requires longer image acquisition time and larger storage space for the data file. Compared to the $100 \mu \mathrm{m}$ pixel pitch, the total effective channel area of $T_{\mathrm{AMP}}$ and $T_{\mathrm{READ}}$ for a $75 \mu \mathrm{m}$ pixel will shrink from $2 \times 30 \mu \mathrm{m} \times$ $50 \mu \mathrm{m}$ to $2 \times 20 \mu \mathrm{m} \times 20 \mu \mathrm{m}$ using the fork-shaped TFT structure. Hence the $W / L$ of $T_{\mathrm{AMP}}$ and $T_{\mathrm{READ}}$ is normalized to $40 \mu \mathrm{m} / 5 \mu \mathrm{m}$. $C_{\mathrm{PD}}$ is around $1 \mathrm{pF}$, which corresponds to the proposed organic photodiode capacitance of a single pixel; ${ }^{25}$ if $C_{\mathrm{PD}}$ is reduced, the gain will increase, but the dynamic range will be reduced.

Figure 6(a) shows the simulated output voltage $\left(V_{\text {OUT }}\right)$ for a-Si:H APS, based on the proposed readout scheme. It can be seen that during the readout time $(20 \mu \mathrm{s})$, a photocurrent $\left(I_{\mathrm{ph}}\right)$ from 0.01 to $0.5 \mathrm{nA}$ can be detected. To optimize the pixel design, different channel widths of $T_{\mathrm{AMP}}$ and $T_{\mathrm{READ}}\left(W_{\mathrm{AMP}}\right.$ and $W_{\text {READ }}$ ) were investigated with the channel length fixed to $5 \mu \mathrm{m}\left(L_{\mathrm{AMP}}=L_{\mathrm{READ}}=5 \mu \mathrm{m}\right)[$ Fig. 6(b)]. The output charge is defined as the variance between the integrated charge on the amplifier under illumination and dark condition. First, when we set $W_{\mathrm{AMP}}=W_{\mathrm{READ}}$ to be 40,30 , and $20 \mu \mathrm{m}$, the results indicate that the charge gain is proportional to the transistor channel width. Second, by considering a constant pixel area of $75 \times 75 \mu \mathrm{m}$, the sum of channel widths of $T_{\mathrm{AMP}}$ and $T_{\mathrm{READ}}$ $\left(W_{\mathrm{AMP}}+W_{\mathrm{READ}}\right)$ is $80 \mu \mathrm{m}$. Charge gain of 3.2, 2.8, and 2.1 is achieved for $W_{\mathrm{AMP}} / W_{\mathrm{READ}}$ equals to $40 / 40,50 / 30$, and $60 / 20$, respectively. The result illustrates that maximum charge gain can be achieved, when $W_{\mathrm{AMP}}$ and $W_{\mathrm{READ}}$ match each other. The maximum detectable charge is $5 \mathrm{pC}$, as determined by $C_{\mathrm{PD}}\left(V_{\mathrm{BIAS}}-V_{\mathrm{REF}}\right)$. The maximum charge gain of the $75 \mu \mathrm{m}$ a-Si:H TFT-based APS is only 3.2, which is insufficient for signal amplification and noise reduction needed for next generation DBT as demonstrated in Sec. 4.B. The small charge gain is mainly due to the low TFT field effect mobility (0.5$1 \mathrm{~cm}^{2} / \mathrm{V} \mathrm{s}$ ). The limitations of low mobility a-Si:H APS are a high electronic noise, a long readout time, and a high dose. The charge gain can be improved by increasing the TFT fieldeffect mobility [Eq. (1)] without affecting the pixel size and image resolution. Thereby, a high mobility TFT technology is required to go beyond a-Si:H TFTs. 
(a)

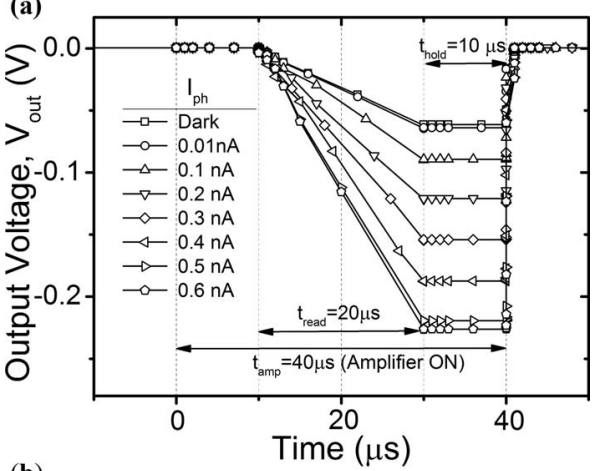

(b)

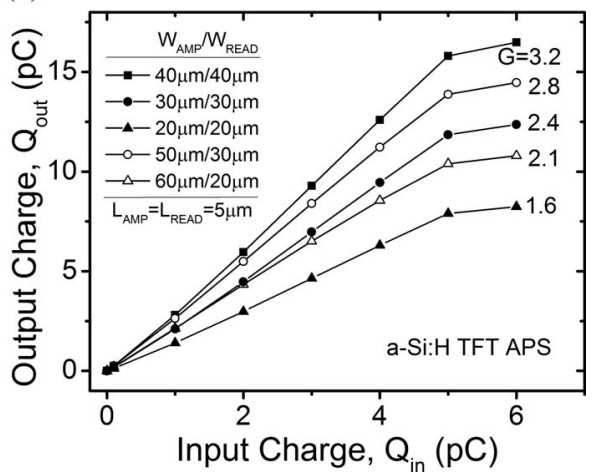

FIG. 6. (a) Simulated a-Si:H TFT APS circuit output voltage under different photocurrent levels; (b) output charge and gain using different channel widths ( $W_{\mathrm{AMP}}$ and $W_{\mathrm{READ}}$ ) of $T_{\mathrm{AMP}}$ and $T_{\mathrm{READ}}$, while the length of the TFTs is constant $\left(L_{\mathrm{AMP}}=L_{\mathrm{READ}}=5 \mu \mathrm{m}\right)$, are shown.

a-IGZO TFT technology is an ideal candidate for next generation medical imaging because of a high field-effect mobility $\left(5-20 \mathrm{~cm}^{2} / \mathrm{V} \mathrm{s}\right)$, a low flicker noise $\left(\alpha_{\mathrm{H}} \sim 1.5 \times 10^{-3}\right)$, a small TFT off-current $\left(<10^{-13} \mathrm{~A}\right)$, and a low-temperature fabrication process. ${ }^{54,55}$ The simulated output-input signal characteristics of a-IGZO TFT-based APS are shown in Fig. 7(a) using the same driving schemes as shown in Fig. 4(b). We change the field-effect mobility value from 5 to $20 \mathrm{~cm}^{2} / \mathrm{V} \mathrm{s}$ [which can be easily realized by amorphous metal-oxide TFTs (Refs. 54-56)] to evaluate the relationship between APS charge gain and TFT mobility. A charge gain of at least 31 is achieved and the maximum value can be above 100 if a large mobility $\left(20 \mathrm{~cm}^{2} / \mathrm{V} \mathrm{s}\right)$ (Ref. 56) of metal oxide TFTs is selected. Further increase in the charge gain leads to saturation of the feedback capacitor $\left(C_{\mathrm{FB}}\right)$ without additional signal detection. The charge gain linearly increases with aIGZO TFT mobility as shown in Fig. 7(b), which is consistent with Eq. (1). It is also found using simulation that if $T_{\mathrm{AMP}}$ is chosen to be operating in the saturation region, the output-toinput charge signal relationship is nonlinear. To maintain the signal linearity, we choose $T_{\mathrm{AMP}}$ to be operated in the linear region. We have already reported a fabricated a-IGZO APS with the charge gain $\sim 25$ (normalized to $\sim 30$ with $75 \mu \mathrm{m}$ pixel pitch).$^{57}$ The a-IGZO TFT mobility of the fabricate APS circuit is $5.6 \mathrm{~cm}^{2} / \mathrm{V}$ s. The normalized experimental data of both a-Si:H (Ref. 25) and a-IGZO TFT APS (Ref. 57) are also plotted in Fig. 7(b). Based on both experimental and simulation results, the charge gain of a-IGZO TFT APS is at least $\times 10$ higher than a-Si:H TFT APS with same device and pixel
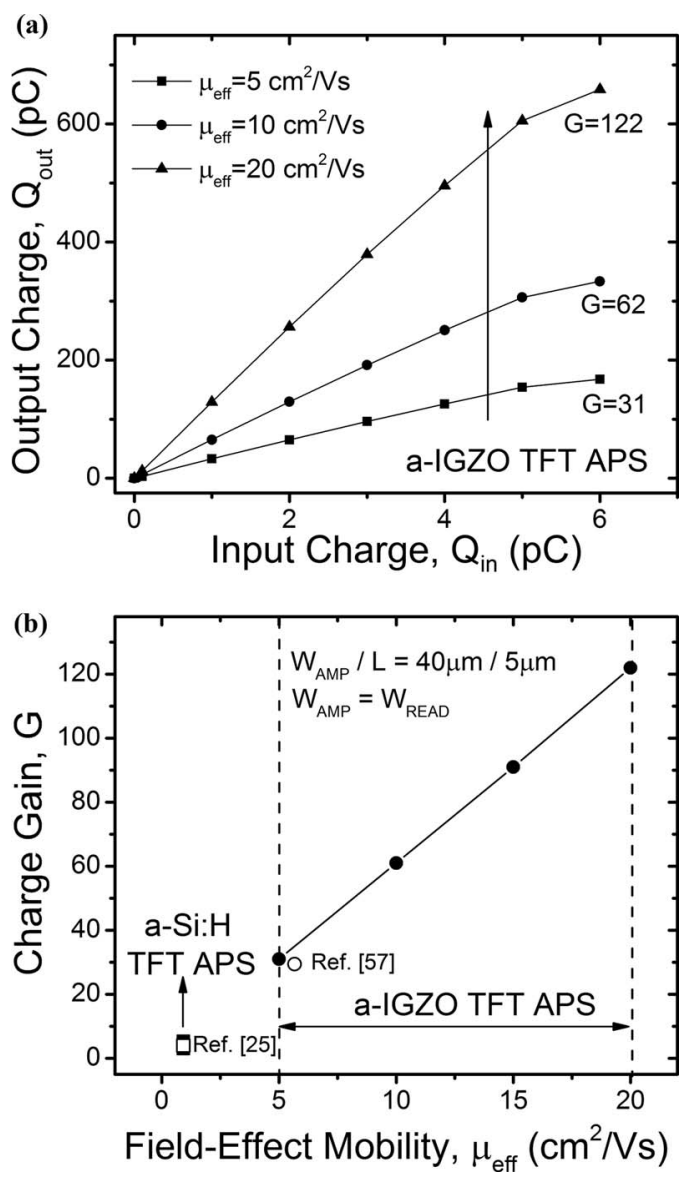

FIG. 7. (a) Simulated integrated charge signal and (b) charge gain for aIGZO TFT active pixel sensor (solid symbol). Field-effect mobility values of the a-IGZO TFTs from 5 to $20 \mathrm{~cm}^{2} / \mathrm{V} \mathrm{s}$ are used. The normalized experimental data (open symbol) of the a-Si:H (Ref. 25) and a-IGZO TFT APS (Ref. 57) are also compared.

dimensions. The large charge gain of a-IGZO APS can minimize the influence of the electronic noise.

In addition to the large charge gain achieved by a-IGZO APS, Fung et al. reported that the low frequency flicker (1/f) noise of a-IGZO TFTs is lower compared to a-Si:H TFTs. ${ }^{55}$ The a-IGZO TFT Hooge's parameter $\left(\alpha_{\mathrm{H}}\right)$ extracted from the $1 / f$ noise measurement is $1.5 \times 10^{-3}$, which is comparable to that of the poly-Si TFTs. ${ }^{58}$ Compared to the $\alpha_{\mathrm{H}}$ value of aSi:H TFTs $\left(3 \times 10^{-3}<\alpha_{\mathrm{H}}<1.2 \times 10^{-2}\right),{ }^{55,59,60}$ the low $\alpha_{\mathrm{H}}$ of a-IGZO TFTs makes them attractive for low-noise applications such as DBT.

Another candidate for APS-based medical imaging system is poly-Si TFT. ${ }^{21,22}$ Detailed comparison of the APS pixel circuit electronic noise using a-Si:H, poly-Si, and a-IGZO TFT technologies is discussed in Sec. 3.C.

\section{C. APS pixel circuit electronic noise}

Each electronic noise element is modeled and calculated based on reported APS noise model developed by Karim et $a l .{ }^{20}$ First, the OPD dark current shot noise is given by ${ }^{49}$

$\sigma_{\mathrm{PD}, \text { shot }}=\sqrt{I_{\mathrm{dark}} t_{\mathrm{frame}} / q}$ 
where $I_{\text {dark }}\left(\sim 4.89 \times 10^{-13} \mathrm{~A}\right)$ is the dark current of OPD (calculated using the dark current density of $10^{-8} \mathrm{~A} / \mathrm{cm}^{2}$, a pixel pitch of $75 \mu \mathrm{m}$, and fill factor of 0.87) and $t_{\text {frame }}$ is the frame time (145 ms).

Similarly, the $T_{\text {RESET }}$ leakage current shot noise is given by $^{49}$

$$
\sigma_{\mathrm{TFT}, \text { shot }}=\sqrt{I_{\mathrm{off}} t_{\mathrm{frame}} / q},
$$

where $I_{\text {off }}$ is the TFT leakage current (off-current). Leakage current of $10^{-12}, 2 \times 10^{-12}$, and $5 \times 10^{-14} \mathrm{~A}$ is used for a$\mathrm{Si}: \mathrm{H}$, poly-Si, and a-IGZO TFTs, respectively. ${ }^{50,54,61}$

If double sampling is implemented, the reset noise for APS is given by ${ }^{49}$

$$
\sigma_{\text {reset }}=\sqrt{2 k T C_{\text {pix }}} / q
$$

where $k$ is the Boltzmann constant, $T$ is the Kelvin temperature, and $C_{\text {pix }}(\sim 1 \mathrm{pF})$ is the input pixel capacitance, which is dominated by $C_{\mathrm{PD}}$.

The input referred preamplifying pixel noise, which cannot be removed by APS, is given by

$$
\sigma_{\text {pre-AMP }}=\sqrt{\sigma_{\mathrm{PD}, \text { shot }^{2}}+\sigma_{\mathrm{TFT}, \text { shot }^{2}}+\sigma_{\text {reset }^{2}}} \text {. }
$$

In this work, since both the $T_{\mathrm{AMP}}$ and $T_{\mathrm{READ}}$ operate in the linear region, the noise power spectral density of thermal noise for $T_{\mathrm{AMP}}$ and $T_{\mathrm{READ}}$ is given by ${ }^{55}$

$$
S_{\text {Thermal }}=4 k T \mu_{\mathrm{eff}} C_{\mathrm{ox}}(W / L)\left(V_{\mathrm{GS}}-V_{\mathrm{T}}\right),
$$

where $k$ is the Boltzmann constant, $T$ is the Kelvin temperature, $C_{\text {ox }}$ is the gate capacitance per unit area $\left(\sim 17 \mathrm{nF} / \mathrm{cm}^{2}\right)$, $W, L$, and $V_{\mathrm{GS}}$ are the channel width, length, and the gate to source voltages of $T_{\mathrm{AMP}}$ and $T_{\mathrm{READ}}$, and $V_{\mathrm{T}}$ is the TFT threshold voltage ( $\sim 5 \mathrm{~V}$ in the simulation). All the driving voltages and device dimension parameters can be found in Table II. The corresponding thermal noise equivalent electrons of $T_{\mathrm{AMP}}$ and $T_{\text {READ }}\left(\sigma_{\text {Thermal,AMP }}\right.$ and $\left.\sigma_{\text {Thermal,READ }}\right)$ can be calculated by integrating the noise power spectral density over the noise bandwidth of a first-order low-pass filtering circuit using the method and equations developed by Karim et al. ${ }^{20}$ The noise calculation for $T_{\mathrm{AMP}}$ is modified for linear region operation.

The noise power spectral density of flicker noise for $T_{\mathrm{AMP}}$ and $T_{\mathrm{READ}}$ operating both in the linear region are given by ${ }^{55}$

$$
S_{\text {Flicker }}=\frac{\alpha_{\mathrm{H}} q \mu_{\mathrm{eff}} C_{\mathrm{ox}} W\left(V_{\mathrm{GS}}-V_{\mathrm{T}}\right) V_{\mathrm{DS}}^{2}}{f L^{3}},
$$

where $\alpha_{\mathrm{H}}$ is the Hooge's parameter $\left(\alpha_{\mathrm{H}}\right.$ equals to $10^{-2}$, $10^{-3}$, and $1.5 \times 10^{-3}$ for a-Si:H, poly-Si, and a-IGZO TFTs, respectively), ${ }^{55,58-60} V_{\mathrm{GS}}$ and $V_{\mathrm{DS}}$ are the gate to source voltage and drain to source voltage of $T_{\mathrm{AMP}}$ and $T_{\mathrm{READ}}$, and $f$ is the frequency. The flicker noise equivalent electrons $\sigma_{\text {Flicker,AMP }}$ and $\sigma_{\text {Flicker,READ }}$ can be calculated by integrating the noise spectral density over frequency adopted from the model developed by Karim et al. ${ }^{20}$

The external readout circuit noise $\left(\sigma_{\mathrm{amp}}\right)$ is mainly generated by the switched-capacitor amplifier. The readout circuit noise can be modeled as having a fixed noise component of the amplifier $\left(\sigma_{\text {amp } 0}\right)$ in addition to capacitance dependent component $\left(\delta C_{\mathrm{d}}\right)$ (Refs. 20 and 62)

$$
\sigma_{\mathrm{amp}}=\sigma_{\mathrm{amp} 0}+\delta C_{\mathrm{d}}
$$

where $\sigma_{\text {amp }}$ and $\delta$ are an amplifier's characteristic constants. $C_{\mathrm{d}}$ is the capacitance loading at the amplifier's input node, which mainly includes the capacitance on the data line, $C_{\text {DATA }}$ [Fig. 4(a)]. For the specific low-noise amplifier, $\sigma_{\text {amp } 0}$ and $\delta$ are found to be $250 e$ and as $15 e / \mathrm{pF}$, respectively. ${ }^{20}$ The dominant contribution to $C_{\text {DATA }}$ is the total parasitic capacitance from the overlap area between each gate line and data line. $C_{\text {DATA }}$ is typically in the order of $100 \mathrm{pF}^{20,62}$

The output referred postamplifying pixel noise is given by

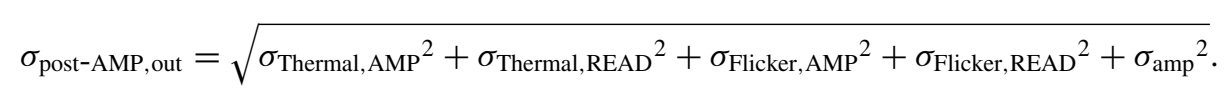

The input referred postamplifying pixel noise, which can be significantly reduced by APS, is given by output referred postamplifying pixel noise divided by the APS charge gain $\left(\sigma_{\text {post-AMP,in }}=\sigma_{\text {post-AMP,out }} / G\right)$. Finally, the total input referred noise is given by the square root of the quadratic sum of the input referred preamplifying and postamplifying noise.

We are comparing the input referred noise equivalent electrons for a-Si:H, poly-Si, and a-IGZO APS in Fig. 8. All the noise elements discussed above are included in the calculation. The photodiode dark current density is $10^{-8} \mathrm{~A} / \mathrm{cm}^{2}$ referring to that of OPD, ${ }^{23}$ which is used for a-Si:H, poly-Si, and a-IGZO APS. The mobility of a-Si:H, poly-Si, and aIGZO TFTs is set to be $0.5,100$, and $10 \mathrm{~cm}^{2} / \mathrm{V} \mathrm{s}$, corresponding to charge gains of 3.3, 664, and 69 for a $75 \mu \mathrm{m}$ pixel pitch, respectively. The calculated charge gain is consistent with that of SPICE simulation. The calculated total input referred noise for a-Si:H, poly-Si, and a-IGZO APS is 2178 , 1618 , and $912 e$, respectively.

First, for a-Si:H APS, as shown in Fig. 8, both the preamplifying noise and postamplifying noise are significant. The preamplifying noise is dominated by the TFT leakage current shot noise of $T_{\text {RESET }}(952 e$ ) due to the high leakage current of $\sim 10^{-12} \mathrm{~A}$. It is demonstrated that the postamplifying noise of a-Si:H APS remains high because of a low field-effect mobility $\left(\sim 0.5 \mathrm{~cm}^{2} / \mathrm{V} \mathrm{s}\right)$ and a low charge gain $(\sim 3)$. Therefore, the total electronic pixel circuit noise of a-Si:H APS is high. The a-Si:H TFT APS cannot be used for DBT because of its low carrier mobility, limited charge gain, and large threshold 


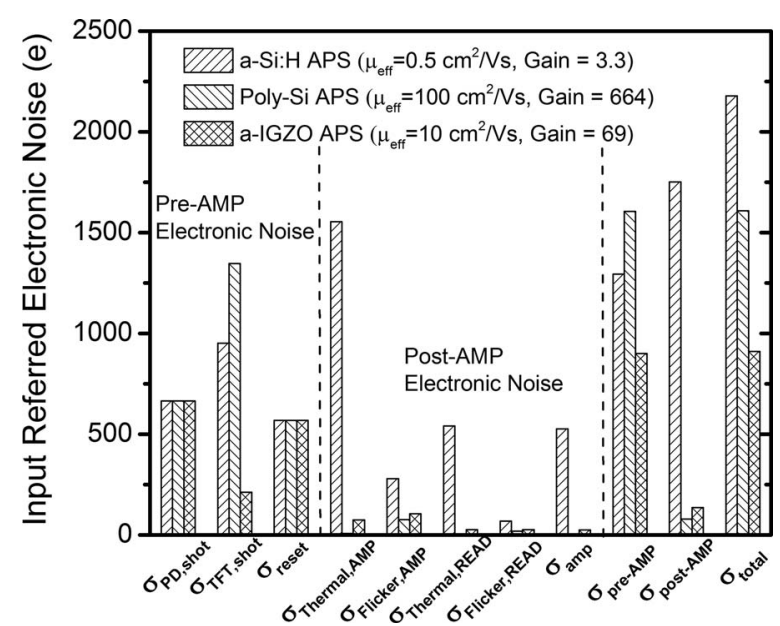

FIG. 8. Calculated input referred noise equivalent electrons of all noise elements for a-Si:H, poly-Si, and a-IGZO APS. The mobility of a-Si:H, poly-Si, and a-IGZO TFTs is set to be $0.5,100$, and $10 \mathrm{~cm}^{2} / \mathrm{V} \mathrm{s}$, corresponding to charge gains of $3.3,664$, and 69 , respectively. Leakage current of $10^{-12}, 2$ $\times 10^{-12}$, and $5 \times 10^{-14} \mathrm{~A}$ are used for a-Si:H, poly-Si, and a-IGZO TFTs, respectively. The photodiode dark current is $10^{-8} \mathrm{~A} / \mathrm{cm}^{2}$, referring to the experimental data of OPD. The frame time is $145 \mathrm{~ms}$.

voltage shift ${ }^{63}$ during operation. Izadi et al. optimized the aSi:H APS using theoretical calculations and reported total input referred noise of $<1000 e .^{64}$ To achieve such low value, a small pixel capacitance of $<100 \mathrm{fF}$ has to be used to achieve a larger charge gain for a-Si:H APS. However, such low capacitance value is not suitable for DBT due to the dominating capacitance of OPD $(\sim 1 \mathrm{pF})$ and will result in a limited dynamic range owning to a small maximum detectable signal. For typical DBT detectors with a pixel capacitance of $\sim 1 \mathrm{pF}$, the optimized a-Si:H TFT dimension is $W / L=100 \mu \mathrm{m} / 5 \mu \mathrm{m} .{ }^{64}$ Therefore, we conclude that a-Si:H APS cannot be used for high resolution DBT due to a large TFT dimension.

For poly-Si APS, the significant charge gain $(\sim 664)$ effectively removes the postamplifying noise. However, a high TFT leakage current $\left(\sim 2 \times 10^{-12} \mathrm{~A}\right)$ results in a high TFT leakage current shot noise (1346e $e$ ), which cannot be removed by APS. Antonuk et al. have demonstrated that there was little improvement in the signal-to-noise properties of poly-Si APS prototype imagers due to their high level of electronic noise. ${ }^{22}$ They claim that the grain boundaries of polysilicon contain numerous trap states, which are associated with a large leakage current (high TFT shot noise), a high $1 / f$ noise, and a poor electrical property (threshold voltage, mobility, and current leakage) uniformity. ${ }^{22}$ The imaging performance of fabricated poly-Si APS array is consistent with our noise modeling results. Because the polycrystalline nature cannot be modified, the poor SNR will prevent poly-Si APS to be used for DBT using existing poly-Si TFT technology.

As shown in Fig. 8, the minimum input referred electronic noise is achieved by a-IGZO APS. For a-IGZO TFTs with a field-effect mobility of $10 \mathrm{~cm}^{2} / \mathrm{V} \mathrm{s}$, the calculated charge gain is around 69. This value is consistent with the SPICE simulation results in Fig. 7. The charge gain is sufficient to eliminate the postamplifying noise (137 e). In addition, the low off-current of a-IGZO TFTs $\left(5 \times 10^{-14} \mathrm{~A}\right)$ (Ref. 48) reduces the preamplifying noise (901e). The total noise is only $912 e$, which is suitable for low-dose DBT. The a-IGZO APS total electronic noise can be further reduced to $658 e$ if the OPD dark current is reduced to $10^{-9} \mathrm{~A} / \mathrm{cm}^{2}$. The APS noise can be optimized when the driving scheme, device structure, and layout are optimized for a given application such as DBT, which is outside of the scope of this paper. Already Sony Corporation demonstrated excellent a-IGZO TFT uniformity and reliability with a low leakage current and noise. ${ }^{65}$ Finally, the low process temperature favors the fabrication of a-IGZO TFTs on flexible or deformable substrates, ${ }^{54,66}$ which is attractive to future flexible $\mathrm{x}$-ray imaging applications. ${ }^{67}$

\section{NOISE AND DETECTIVE QUANTUM EFFICIENCY}

\section{A. Cascaded system model}

To analyze the electrical performance such as noise and DQE of the a-IGZO APS AMFPI, the cascaded system analysis ${ }^{18,30-32,36}$ is an effective and reliable approach. The model was described in detail by Siewerdsen et al. by considering the noise transfer as gain and spreading stages. ${ }^{18}$ For a gain stage, the noise power spectra of stage $i\left(S_{i}\right)$ as a function of spatial frequency is determined by the average gain $\left(\bar{g}_{i}\right)$, the mean fluence of input quanta $\left(\overline{q_{i-1}}\right)$, gain variance $\left(\sigma_{g i}\right)$, and additional electronic noise element in the stage $\left(S_{\text {add }}\right),{ }^{18}$

$$
S_{i}(u, v)={\overline{g_{i}}}^{2} S_{i-1}(u, v)+\sigma_{g_{i}}^{2} \overline{q_{i-1}}+S_{\mathrm{add}_{i}}(u, v) .
$$

The spreading stages represent the image blurring due to input quanta spreading over the spatial frequency in two dimensions. For a stochastic spreading stage, the input signal is blurred due to light scattering. This is the case for the scintillator scattering effect. The noise transfer for a stochastic spreading stage is ${ }^{18}$

$$
S_{i}(u, v)=\left[S_{i-1}(u, v)-\overline{q_{i-1}}\right] T_{i}^{2}(u, v)+\overline{q_{i-1}},
$$

where $\overline{q_{i-1}}$ represents the uncorrelated component that is not affected by the spreading stage, and $T_{i}(u, v)$ is the MTF of the $i$ th stage. On the other hand, for a deterministic spreading stage, the NPS is directly blurred by a MTF, ${ }^{18}$

$$
S_{i}(u, v)=S_{i-1}(u, v) T_{i}^{2}(u, v) .
$$

The deterministic spreading corresponds to the image blurring by the presampling pixel MTF in the fifth stage, $T_{5}(u, v)$, in the tomosynthesis system. The complete noise transfer of the system can be described by a series of gain and spreading stages modeled by the cascaded system analysis. The flow chart of the cascaded system is shown in Fig. 9.

The APS amplifies both the quantum noise and the preamplifying electronic noise by the charge gain $\left(\bar{g}_{6}\right){ }^{20}$ Thus, the output referred NPS as a function of spatial frequency of the entire DBT system in the unit of $e^{2} \mathrm{~mm}^{2}$ can be derived to be $^{18}$

$$
\begin{aligned}
S(u, v)= & \left(a_{\mathrm{pd}}^{4} \overline{q_{0} g_{1} g_{2} g_{4}}\left[1+\overline{g_{4}}\left(\overline{g_{2}}+\varepsilon_{g_{2}}\right) T_{3}^{2}(u, v)\right]\right. \\
& \left.\times T_{5}^{2}(u, v)+S_{\text {pre-AMP }}(u, v)\right){\overline{g_{6}}}^{2} \\
& +S_{\text {post-AMP }}(u, v),
\end{aligned}
$$




\begin{tabular}{l}
\hline Stage 0: Incident X-ray quanta \\
$\begin{array}{l}\text { Stage 1: Interaction of incident X-ray quanta with } \\
\text { the scintillator (gain stage) }\end{array}$ \\
\begin{tabular}{|l|}
\hline Stage 3: Scattering effect of the scintillator \\
(stochastic spreading stage)
\end{tabular} \\
\begin{tabular}{|l|} 
Stage 4: Coupling of the optical photons to the \\
photodiode (gain stage)
\end{tabular} \\
\begin{tabular}{|l|} 
Stage 5: Integration of photons by the square pixel \\
aperture (deterministic spreading stage)
\end{tabular} \\
\begin{tabular}{|l} 
Stage 6: Signal amplification by the APS pixel \\
circuit (gain stage)
\end{tabular} \\
\hline
\end{tabular}

FIG. 9. A seven-step cascaded system flow chart showing the signal and noise transfer of APS indirect $\mathrm{x}$-ray imager.

where $a_{\mathrm{pd}}$ is photodiode active area considering the pixel fill factor, $\bar{g}_{i}$ and $T_{i}$ are the gain and MTF of stage $i, \varepsilon_{g_{2}}$ is the Poisson excess of gain stage $2, S_{\text {pre-AMP }}$ and $S_{\text {post-AMP }}$ are the output referred NPS of preamplifying and postamplifying pixel electronic noise, respectively. Both the quantum noise (photon shot noise) and electronic noise are included in the model. The quantum noise is given by the first term in Eq. (14). The output referred individual pixel variance of the system can be obtained by integrating the NPS over the spatial frequency,

$$
\begin{aligned}
\sigma_{\text {tot }}^{2}= & \left(\iint_{u} a_{\mathrm{pd}}^{4} \overline{q_{0} g_{1} g_{2} g_{4}}\left[1+\overline{g_{4}}\left(\overline{g_{2}}+\varepsilon_{g_{2}}\right) T_{3}^{2}(u, v)\right]\right. \\
& \left.\times T_{5}^{2}(u, v) d u d v+\sigma_{\text {pre-AMP }}^{2}\right){\overline{g_{6}}}^{2}+\sigma_{\text {post-AMP }}^{2}
\end{aligned}
$$

The MTF of the system is given by

$$
T(u, v)=T_{3}(u, v) T_{5}(u, v),
$$

where $T_{3}$ represents the MTF due to the scintillator scattering effect and $T_{5}$ is the MTF associated with the pixel aperture. We have ${ }^{18}$

$$
\begin{aligned}
& T_{3}(u, v) \approx \frac{1}{1+H \cdot\left(u^{2}+v^{2}\right)}, \\
& T_{5}(u, v)=\left|\operatorname{sinc}\left(a_{\mathrm{pd}} u\right) \cdot \operatorname{sinc}\left(a_{\mathrm{pd}} v\right)\right|,
\end{aligned}
$$

where $H$ is a parameter describing the scattering effect of the scintillator.

The DQE of the system is determined by the square ratio of the output SNR to the input SNR. The DQE of the proposed APS x-ray imager is

$$
\begin{aligned}
\operatorname{DQE}(u, v) & =\frac{\left(\mathrm{SNR}_{\mathrm{out}}\right)^{2}}{\left(\mathrm{SNR}_{\mathrm{in}}\right)^{2}}=\frac{\overline{d^{2}} \cdot T^{2}(u, v)}{\overline{q_{0}} \cdot S(u, v)} \\
& =\frac{a_{\mathrm{pd}}^{4} \overline{q_{0}}\left[\overline{g_{1} g_{2} g_{4} g_{6}} \cdot T(u, v)\right]^{2}}{S(u, v)},
\end{aligned}
$$

TABLE III. Key parameters used in the cascaded system model.

\begin{tabular}{lcc}
\hline \hline Parameters & Value & Description \\
\hline $\mathrm{X}$ & $10 \mu \mathrm{R}$ to $10 \mathrm{mR}$ & Exposure \\
$\bar{q}_{0} / X$ & 46520 (Unit: x rays $\left./ \mathrm{mm}^{2} / \mathrm{mR}\right)$ & Mean x-ray fluence \\
$\bar{g}_{1}$ & 0.83 & Gain of stage 1 \\
$\bar{g}_{2}$ & 520 & Gain of stage 2 \\
$\varepsilon_{\mathrm{g} 2}$ & 5.26 & Poisson excess of stage 2 \\
$H$ & 0.18 & Scattering parameter of stage 3 \\
$\bar{g}_{4}$ & 0.6 & Gain of stage 4 \\
$\bar{g}_{6}$ & $3.3-664$ & APS charge gain of stage 6 \\
$a_{\text {pix }}$ & $50-125 \mu \mathrm{m}$ & Pixel pitch \\
$F F$ (APS) & 0.87 (vertically stacked) & APS pixel fill factor \\
$F F$ (PPS) & 0.45 (in-plane) & PPS pixel fill factor \\
\hline \hline
\end{tabular}

where $\mathrm{SNR}_{\text {out }}$ and $\mathrm{SNR}_{\text {in }}$ are the output and input signal-tonoise ratio, respectively, $\bar{d}$ is the mean output signal in unit of electrons, $\overline{q_{0}}$ is the incident x-ray fluence, and $T$ [Eq. (16)] and $S$ [Eq. (14)] are the MTF and NPS of the system, respectively. The output electrons can be converted to voltage, which is then converted to a digital number (output digital signal). The results of the noise and DQE modeling using the cascaded system are shown in Sec. 4.B-4.D. All the key parameters associated with the cascaded system model are listed in Table III. The parameters associated with the x-ray source and the scintillator were reported by El-Mohri et al. ${ }^{36}$ corresponding to a $26 \mathrm{kVp}$ incident $\mathrm{x}$-ray beam and high light output (HL) CsI:Tl scintillator ${ }^{46}$ used for mammography and tomosynthesis. The scintillator deposited on a fiber optic plate was used to extract those parameters. The scintillator gain of the HL CsI:Tl is limited by the low optical transmission $(\sim 60 \%)$ of the fiber optic plate. ${ }^{36}$ Direct deposition of the CsI:Tl scintillator on top of the OPD will achieve a high scintillator gain of $\sim 520$ photons/x-ray $\left(\bar{g}_{2}\right)$. The APS charge gain of stage 6 is 3.3, 69, and 664 for a-Si:H, a-IGZO, and poly-Si APS, respectively. The pixel $F F$ using the vertically stacked OPD/APS TFT backplane structure is 0.87 , while the $F F$ of conventional in-plane PPS is $\sim 0.45$ for a $75 \mu \mathrm{m}$ pixel pitch.

\section{B. Noise of the a-IGZO active pixel sensor imager}

APS pixel circuit is proposed to reduce the total pixel noise, which contains both the quantum noise (photon shot noise) and electronic noise. The quantum noise and the preamplifying APS electronic noise cannot be eliminated by APS, while the postamplifying noise can be minimized. Figure 10 shows the input referred total pixel noise of the proposed AMFPI with a pixel pitch of $75 \mu \mathrm{m}$ as a function of exposure. The same OPD is used for all devices. The calculation is based on the cascaded system [Eq. (15)] discussed in Sec. 4.A. The input referred noise variance is calculated by the output noise divided by the charge gain. The detector entrance exposure range is from $10 \mathrm{mR}$, which is an average of mammography exposure, to $10 \mu \mathrm{R}$, which is comparable to the exposure of normal fluoroscopy. ${ }^{62}$ The general exposure of tomosynthesis is around $1 \mathrm{mR} .{ }^{62} \mathrm{We}$ pushed the lower end of exposure to $0.1 \mathrm{mR}$ to investigate the 


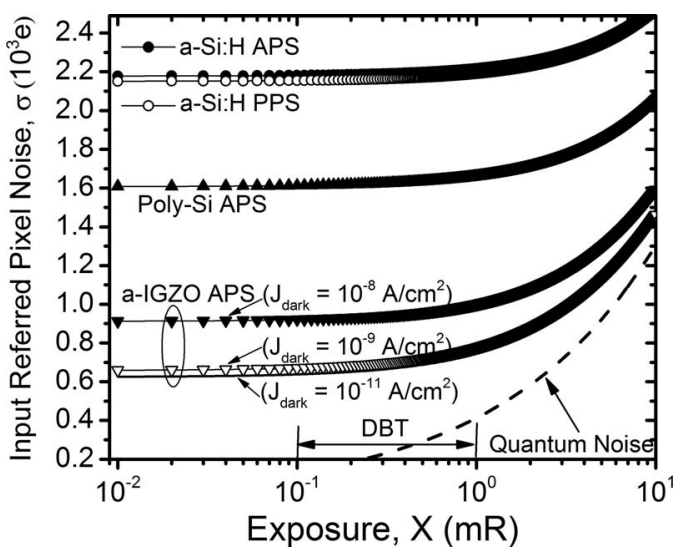

FIG. 10. Input referred pixel noise of $75 \mu \mathrm{m}$ a-Si:H, poly-Si, and a-IGZO APS AMFPIs under exposures ranging from $10 \mu \mathrm{R}$ to $10 \mathrm{mR}$. The a-Si:H PPS imager data are also shown. Both the quantum noise (dashed line) and electronic noise are included. The pixel noise of the a-IGZO APS imager can be further reduced by reducing the dark current of OPD from $10^{-8}$ to below $10^{-9} \mathrm{~A} / \mathrm{cm}^{2}$.

feasibility of reducing the patient dose during tomosynthesis. The performance of a-Si:H, poly-Si, and a-IGZO APS AMFPIs is compared with that of conventional a-Si:H PPS AMFPI. The calculated electronic noise elements of a-Si:H, poly-Si, and a-IGZO APS imagers, as shown in Fig. 8, are integrated into the cascaded system modeling. The result shows that the quantum noise is dominant for a high exposure ( $>1 \mathrm{mR})$ condition such as mammography. For a low exposure $(<1 \mathrm{mR})$ condition for DBT, the noise is dominated by the electronic noise. The total noise of a-Si:H APS imager is comparable to that of the a-Si:H PPS imager because of its low carrier mobility, which is not sufficient to reduce the APS postamplifying electronic noise. The poly-Si TFT leakage current results in a high preamplifying APS noise, which cannot be eliminated. The a-IGZO APS imager shows a superior noise performance compared to a-Si:H and poly-Si APS because of a high TFT mobility (high charge gain) and a low TFT leakage current. Therefore, the proposed a-IGZO APS is suitable for low dose DBT. The APS noise can be further reduced, if the dark current of OPD can be reduced to by one order of magnitude. As shown in Fig. 7, the reset noise becomes dominant if the OPD dark current is minimized. Based on this analysis, OPD dark current in the range of $10 \mathrm{pA} / \mathrm{cm}^{2}$ to $1 \mathrm{nA} / \mathrm{cm}^{2}$ is required for DBT. In comparison, the a-Si ITO/p-i-n/Mo photodiode dark current density is about $40 \mathrm{pA} / \mathrm{cm}^{2}(@-1 \mathrm{~V})$.

\section{C. Detective quantum efficiency of a-IGZO active pixel sensor imager}

DQE is calculated using Eq. (19) to analyze the detector performance. The small pixel size $(75 \mu \mathrm{m})$, small pixel fill factor (0.45), low tomosynthesis $\mathrm{x}$-ray exposure, and large electronic noise result in a low DQE for conventional a-Si:H PPS imager [Fig. 11(a)]. The DQE of PPS imager is vanishing especially for higher spatial frequency $(>5 \mathrm{lp} / \mathrm{mm})$. The issue of a low DQE for PPS imager caused by a low SNR can be solved by replacing the PPS by the APS imager. We compare the DQE of a-Si:H, poly-Si, and a-IGZO APS AMFPIs using
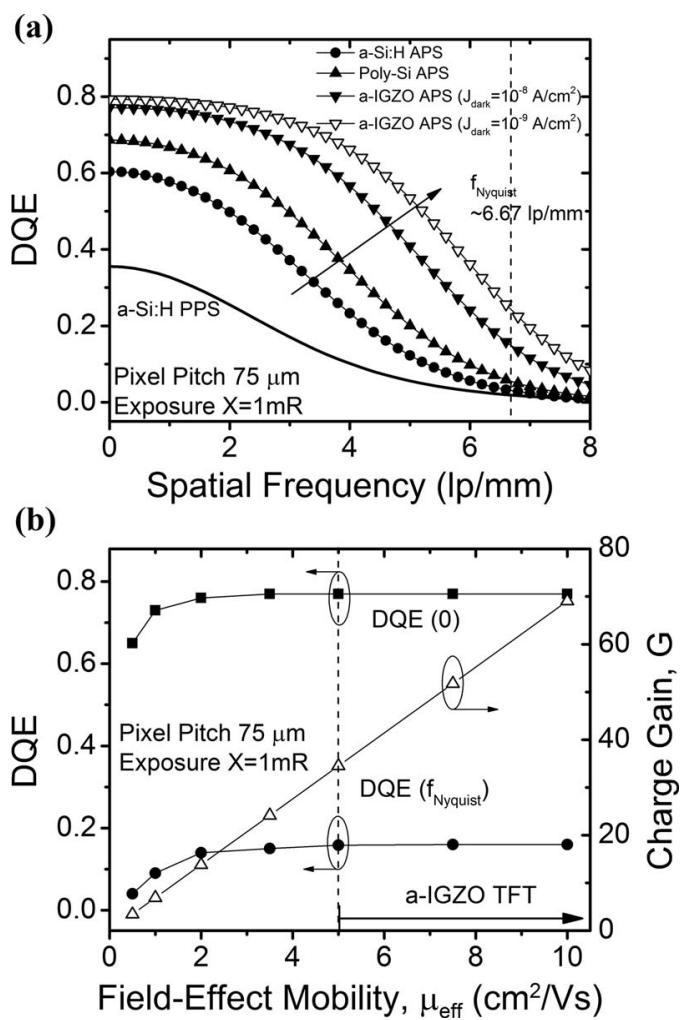

FIG. 11. (a) Calculated DQE vs spatial frequency for a-Si:H PPS, a-Si:H, poly-Si, and a-IGZO APS x-ray imagers. The influence of OPD dark current on DQE is investigated. (b) DQE vs TFT field-effect mobilities at zero spatial frequency $[\mathrm{DQE}(0)]$ and Nyquist frequency $\left[\mathrm{DQE}\left(f_{\text {Nyquist }}\right)\right]$ are shown. The APS charge gain variation for TFT field-effect mobility from 0.5 to $10 \mathrm{~cm}^{2} / \mathrm{V} \mathrm{s}$ is also shown.

the same OPD vertically stacked on top of the TFT backplane resulting in a $F F$ of 0.87 . The proposed a-IGZO APS imager shows excellent $\mathrm{DQE}$ values at both zero spatial frequency $[\mathrm{DQE}(0)]$ and at Nyquist frequency $\left[\mathrm{DQE}\left(f_{\text {Nyquist }}\right)\right]$ due to its low pixel noise $(<1000 e)$ and significant signal. From Fig. 11(b), DQE $(0)$ and DQE $\left(f_{\text {Nyquist }}\right)$ both increase with TFT field-effect mobility. Both $\mathrm{DQE}(0)$ and $\mathrm{DQE}\left(f_{\text {Nyquist }}\right)$ saturate if TFTs with field-effect mobility of $5 \mathrm{~cm}^{2} / \mathrm{V} \mathrm{s}$ are used. This can be easily achieved using proposed a-IGZO TFTs. The corresponding charge gain for $\mu_{\mathrm{eff}}=5 \mathrm{~cm}^{2} / \mathrm{V} \mathrm{s}$ is $\sim 30$ as shown by both simulation and experimental ${ }^{57}$ results. $\mathrm{DQE}\left(f_{\mathrm{Nyquist}}\right)$ is around 0.2 , which demonstrates that the Nqyuist frequency of $\sim 6.67 \mathrm{lp} / \mathrm{mm}$ can be resolved using a $75 \mu \mathrm{m}$ a-IGZO APS $\mathrm{x}$-ray imaging system. The imaging performance can be further improved if the dark current of OPD can be reduced. The high DQE for spatial frequencies from zero to $f_{\text {Nyquist }}$ makes a-IGZO APS an excellent candidate for next generation high resolution DBT imagers.

The limiting resolution of detectors is determined by the Nyquist frequency that is influenced by the pixel size. The Nyquist frequency can be improved from $\sim 5$ to $\sim 6.67 \mathrm{lp} / \mathrm{mm}$, if a $75 \mu \mathrm{m}$ APS pixel pitch is used. To further improve the resolution to $\sim 10 \mathrm{lp} / \mathrm{mm}$, the pixel size has to be decreased to $50 \mu \mathrm{m}$. The calculated MTFs with different pixel pitch are shown in Fig. 12(a). The MTF at higher spatial frequency improves for smaller pixel size. Both the scintillator blurring 


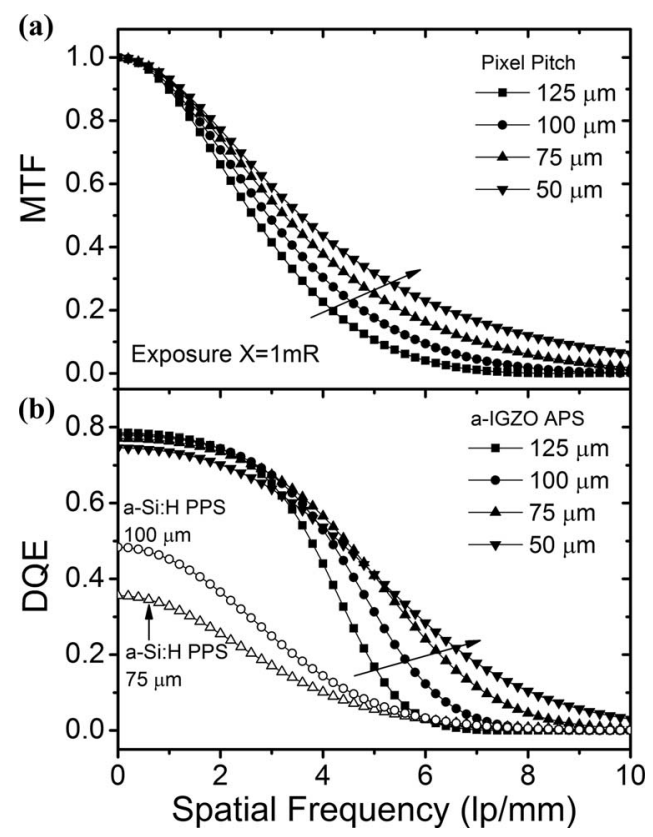

FIG. 12. Calculated MTF (a) and DQE (b) for a-IGZO APS $\left(\mu_{\text {eff }}=\right.$ $10 \mathrm{~cm}^{2} / \mathrm{V} \mathrm{s}$ ) imager vs spatial frequency with pixel pitch ranging from 50 to $125 \mu \mathrm{m}$. Obtained results are compared with the DQE of a-Si:H PPS imager.

effect $\left[T_{3}\right.$ as in Eq. (17)] and the pixel aperture effect $\left[T_{5}\right.$ as in Eq. (18)] are considered in the calculation. The DQE under high spatial frequency for a-IGZO APS imager is plotted in Fig. 12(b). Obtained results demonstrate that the image resolution of a-IGZO APS imager can be improved by reducing the pixel pitch. The Nyquist frequency is $\sim 10 \mathrm{lp} / \mathrm{mm}$ for a $50 \mu \mathrm{m}$ pixel pitch. A pixel size of 75 or $50 \mu \mathrm{m}$ is recommended for DBT. However, smaller pixel pitch requires smaller line width, which could affect large area imagers. It could be difficult to realize a large $W / L$ within a small pixel area $(50 \times 50 \mu \mathrm{m})$, which will result in a limited charge gain. In this case, a high mobility TFT technology such as aInSnZnO (a-ITZO) in combination with advanced TFT structure such as half-Corbino or dual-gate TFT can be used to realize a large charge gain within a small pixel area. ${ }^{56}$ On the contrary, simply shrinking the PPS pixel size will not increase the imager resolution. This is due to a high electronic noise and a small pixel fill factor. Therefore, DQE (0) will be reduced with no improvement in resolution of PPS imager with smaller pixel size. Therefore, we can conclude that spatial frequency above $5 \mathrm{lp} / \mathrm{mm}$ cannot be resolved using the conventional PPS imager.

\section{D. Dose reduction in DBT}

Today the MGD during tomosynthesis is still high (0.67$3.52 \mathrm{mGy}$ ) (Ref. 6) and needs to be reduced. The dose can be reduced by reducing the patient $\mathrm{x}$-ray exposure. At the same time, the detector entrance exposure (x-ray impinging the detector surface after travelling through the patient) will also be reduced accordingly. We explore the a-Si:H PPS and a-IGZO APS imaging performance under a low detector entrance exposure $(<1 \mathrm{mR})$. In Fig. 13(a), DQE variation of APS AMFPI

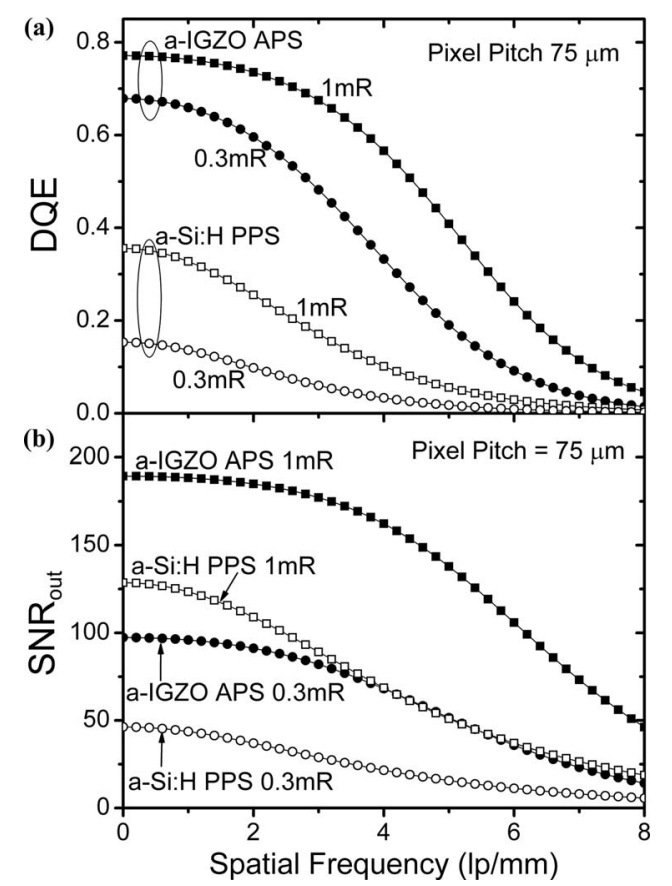

FIG. 13. Calculated DQE (a) and output SNR (b) for a-IGZO APS and aSi:H PPS imagers vs spatial frequency under detector entrance exposure from 0.3 to $1 \mathrm{mR}$.

for $\mathrm{x}$-ray exposure ranging from 0.1 to $1 \mathrm{mR}$ is shown. The field-effect mobility of a-IGZO is $10 \mathrm{~cm}^{2} / \mathrm{V}$ s. For comparison purpose, the data for a-Si:H PPS are also shown. The current DBT exposure $(\sim 1 \mathrm{mR})$ can be reduced to $0.3 \mathrm{mR}$ without a significant reduction of the DQE using the a-IGZO APS imager. The DQE of a-IGZO APS imager under a low exposure $(0.3 \mathrm{mR})$ is at least $2 \times$ greater than that of a-Si PPS imager under a standard DBT exposure (1 mR). The image quality of $\mathrm{x}$-ray imager is determined by the output signal-to-noise ratio $\left(\mathrm{SNR}_{\text {out }}\right)$ as shown in Eq. (13). Figure 13(b) shows that the $\mathrm{SNR}_{\text {out }}$ of the proposed APS imager under $0.3 \mathrm{mR}$ is comparable to that of conventional PPS imager at a much higher $\mathrm{x}$ ray exposure $(1 \mathrm{mR})$ indicating nondegraded image quality at low dose. In combination with recent advances in reconstruction method to increase the $\mathrm{SNR}_{\text {out }},{ }^{68}$ we expect that the patient dose can be reduced by threefold from $\sim 1.3 \mathrm{mGy}$ (avg.) to $0.4 \mathrm{mGy}$ for proposed $\mathrm{x}$-ray imager. However, the dose cannot be reduced using the a-Si:H PPS based x-ray detector because of a poor DQE and $\mathrm{SNR}_{\text {out }}$ at low dose $(<1 \mathrm{mR})$.

\section{DISCUSSION AND CONCLUSION}

Conventional a-Si:H TFT PPS is suitable for mammography due to a high $\mathrm{x}$-ray exposure $(10 \mathrm{mR})$ for a single projection. However, for the multiprojection DBT, the average exposure for a single projection is reduced to around $1 \mathrm{mR}$ to keep overall exposure comparable to mammography. The electronic noise is significant for a small pixel size and a low dose conditions. To detect the small signal, the pixel size is limited to around $100 \mu \mathrm{m}$ and cannot be further reduced. Moreover, the x-ray exposure for current DBT products is high, which leads to a high patient dose during tomosynthesis. 
The merit of the APS pixel architecture is the signal amplification on the pixel level. In this paper, for the first time, a novel high-resolution low-dose APS x-ray imager based on organic photodetector in combination with a-IGZO APS is proposed for DBT. The APS array is vertically stacked to increase pixel fill factor. A high light output CsI:Tl scintillator is also proposed for indirect conversion detection. SPICE circuit simulation is used to analyze the electronic properties of the $75 \mu \mathrm{m}$ APS pixel circuit. The TFT dimensions $(W / L)$ for $T_{\mathrm{RESET}}, T_{\mathrm{AMP}}$, and $T_{\mathrm{READ}}$ are $25 / 5,40 / 5$, and $40 \mu \mathrm{m} / 5 \mu \mathrm{m}$, respectively. We employed the RPI a-Si:H TFT SPICE modified model to simulate the APS circuit based on a-Si:H TFTs and a-IGZO TFTs. The SPICE parameters of a-IGZO TFT were extracted beforehand by fitting the measured TFT characteristics. The simulation results illustrate that charge gains of 3.2 and at least 31 are achieved for a-Si:H and a-IGZO TFT APS, respectively. The charge gain is found to be proportional to the TFT field-effect mobility. Hence, compared to a low mobility $\left(0.5-1 \mathrm{~cm}^{2} / \mathrm{V} \mathrm{s}\right)$ a-Si:H TFTs, a high mobility $\left(5-10 \mathrm{~cm}^{2} / \mathrm{V} \mathrm{s}\right)$ a-IGZO TFTs are ideal to achieve a sufficient charge gain (>30) for DBT application.

The noise and DQE of the entire proposed system are analyzed using cascaded system model by taking into account an additional APS gain stage (stage 6 as in Fig. 9). The pixel pitch is chosen to be $75 \mu \mathrm{m}$ to resolve a high spatial frequency $(\sim 6.67 \mathrm{lp} / \mathrm{mm})$. Both quantum noise and electronic noise are considered. The electronic noise is the dominant factor for a low dose DBT. APS eliminates the TFT flicker noise, readout noise, and external readout circuit noise. Compared with a-Si:H and poly-Si, a-IGZO APS shows a much lower total input referred electronic noise of $<1000 e$. The image quality and resolvability of the proposed $\mathrm{x}$-ray imager is reflected through DQE. Based on the cascaded system modeling, compared to PPS, the DQE of APS imager improves dramatically under both low frequency [DQE (0)] and high frequency $\left[\mathrm{DQE}\left(f_{\text {Nyquist }}\right)\right]$. The a-IGZO with a field-effect mobility $>5 \mathrm{~cm}^{2} / \mathrm{V}$ s shows a superior imaging performance to a-Si:H and poly-Si APS due to a low electronic noise. The limiting Nyquist frequency is determined by the pixel size. Shrinking the pixel size is an effective approach to improve the resolvability of APS systems, while this is not possible to realize for PPS systems due to the dominant electronic noise. The Nyquist frequency of $6.67 \mathrm{lp} / \mathrm{mm}$ is achieved for a $75 \mu \mathrm{m}$ APS pixel. Further reducing the pixel pitch to $50 \mu \mathrm{m}$ will push the Nyquist frequency close to $10 \mathrm{lp} / \mathrm{mm}$. Most importantly, we investigated the x-ray imager performance under a low exposure condition. The current DBT detector entrance exposure is reduced from 1 to $0.3 \mathrm{mR}$. The result shows that the APS x-ray imager under exposure of $0.3 \mathrm{mR}$ still shows a good imaging performance with DQE (0) greater than 0.6, which is at least twice larger than the DQE for a-Si:H PPS under $1 \mathrm{mR}$ exposure. The $S N R_{\text {out }}$ of a-IGZO APS imager is comparable to that of a-Si:H PPS x-ray imager, even though we reduced the x-ray exposure of the a-IGZO APS x-ray imager by threefold (from 1 to $0.3 \mathrm{mR}$ ). Hence, we expect a threefold dose reduction of DBT systems; from avg. $1.3 \mathrm{mGy}$ to less than $0.4 \mathrm{mGy}$. We plan to fabricate the OPD/a-IGZO APS prototype imager in our laboratory through active collab- oration with the industry in near future to verify experimentally presented work in this paper. The results described in this paper show the feasibility and great potential for a-IGZO APS x-ray imager to be used for a high resolution and a low dose DBT.

\section{ACKNOWLEDGMENTS}

The authors would like to thank Dr. John Eric Tkaczyk (GE), Dr. Aldo Badano (FDA), and Professor Mitchell Goodsitt, University of Michigan (Medical School) for the continuous discussions and advices on this work. The authors would also like to thank Dr. Gwanghyeon Baek for the initial APS TFT array mask design and Dr. Tze-Ching Fung for initial a-IGZO TFT APS electronic noise estimation.

a) Author to whom correspondence should be addressed. Electronic mail: kanicki@eecs.umich.edu

${ }^{1}$ R. E. Bird, T. W. Wallace, and B. C. Yankaskas, "Analysis of cancers missed at screening mammography," Radiology 184, 613-617 (1992).

${ }^{2}$ L. T. Niklason et al., "Digital tomosynthesis in breast imaging," Radiology 205, 399-406 (1997).

${ }^{3}$ T. Mertelmeier, J. Orman, W. Haerer, and M. K. Dudam, "Optimizing filtered backprojection reconstruction for a breast tomosynthesis prototype device," Proc. SPIE 6142, 61420F (2006).

${ }^{4}$ Y.-H. Hu, B. Zhao, and Wei Zhao, "Image artifacts in digital breast tomosynthesis: Investigation of the effects of system geometry and reconstruction parameters using a linear system approach," Med. Phys. 35, 5242-5252 (2008).

${ }^{5}$ C. Ghetti, A. Borrini, O. Ortenzia, R. Rossi, and P. L. Ordonez, "Physical characteristics of GE Senographe Essential and DS digital mammography detectors," Med. Phys. 35, 456-463 (2008).

${ }^{6}$ S. S. J. Feng and I. Sechopoulos, "Clinical digital breast tomosynthesis system: Domestic characterization," Radiology 263, 35-42 (2012).

${ }^{7}$ B. Zhao and W. Zhao, "Imaging performance of an amorphous selenium digital mammography detector in a breast tomosynthesis system," Med. Phys. 35, 1978-1987 (2008).

${ }^{8}$ E. Shaheen, N. W. Marshall, and H. Bosmans, "Investigation of the effect of tube motion in breast tomosynthesis: Continuous or step and shoot?," SPIE Med. Imaging 7961, 79611E (2011).

${ }^{9}$ J. A. Baker and J. Y. Lo, "Breast tomosynthesis: State-of-the-art and review of the literature," Acad. Radiol. 18, 1298-1310 (2011).

${ }^{10}$ I. Sechopoulos, "A review of breast tomosynthesis. Part I. The image acquisition process," Med. Phys. 40, 014301 (12pp.) (2013).

${ }^{11}$ S. Richard and E. Samei, "Quantitative breast tomosynthesis: From detectability to estimability," Med. Phys. 37, 6157-6165 (2010).

12 A. S. Chawla et al., "Optimized image acquisition for breast tomosynthesis in projection and reconstruction space," Med. Phys. 36, 4859-4869 (2009).

${ }^{13}$ R. J. Acciavatti and A. D. A. Maidment, "Investigating the potential for super-resolution in digital breast tomosynthesis," Proc SPIE 7961, 79615K (2011).

${ }^{14}$ M. Spahn, "Flat detectors and their clinical applications," Eur. Radiol. 15, 1934-1947 (2005).

${ }^{15}$ L. E. Antonuk, Y. El-Mohri, A. Hall, K.-W. Jee, M. Maolinbay, S. C. Nassif, X. Rong, J. H. Siewerdsen, Q. Zhao, and R. L. Weisfield, "Large-area $97 \mu \mathrm{m}$ pitch indirect-detection active-matrix flat-panel imager (AMFPI)," Proc. SPIE 3336, 2-13 (1998).

${ }^{16}$ R. L. Weisfield, M. A. Hartney, R. A. Street, and R. B. Apte, "New amorphous-silicon image sensor for X-ray diagnostic medical imaging applications," Proc. SPIE 3336, 444-452 (1998).

${ }^{17} \mathrm{~S}$. Vedantham et al., "Full breast digital mammography with an amorphous silicon-based flat panel detector: Physical characteristics of a clinical prototype," Med. Phys. 27, 558-567 (2000).

${ }^{18}$ J. H. Siewerdsen, L. E. Antonuk, Y. El-Mohri, J. Yorkston, W. Huang, and J. M. Boudry, "Empirical and theoretical investigation of the noise performance of indirect detection, active matrix flat-panel imagers (AMFPIs) for diagnostic radiology," Med. Phys. 24, 71-89 (1997).

${ }^{19}$ J. T. Dobbins III, "Tomosynthesis imaging: At a translational crossroads," Med. Phys. 36, 1956-1967 (2009). 
${ }^{20}$ K. S. Karim, A. Nathan, and J. A. Rowlands, "Amorphous silicon active pixel sensor readout circuit for digital imaging," IEEE Trans. Electron Dev. 50, 200-208 (2003)

${ }^{21}$ M. Koniczek, Y. El-Mohri, L. E. Antonuk, A. Liang, Q. Zhao, and H. Jiang, "Noise performance limits of advanced X-ray imagers employing poly-Sibased active pixel architectures," Proc. SPIE 7961, 79610P (2011).

${ }^{22}$ Y. El-Mohri, L. E. Antonuk, M. Koniczek, Q. Zhao, Y. Li, R. A. Street, and J.-P. Lu, "Active pixel imagers incorporating pixel-level amplifiers based on polycrystalline-silicon thin-film transistors," Med. Phys. 36, 3340-3355 (2009).

${ }^{23}$ F. Arca, E. Kohlstädt, S. F. Tedde, P. Lugli, and O. Hayden, "Large active area organic photodiodes for short-pulse X-ray detection," IEEE Trans. Electron Dev. 60, 1663-1667 (2013).

${ }^{24}$ S. F. Tedde, J. Kern, T. Sterzl, J. Fürst, P. Lugli, and O. Hayden, "Fully spray coated organic photodiodes," Nano Lett. 9, 980-983 (2009).

${ }^{25}$ S. F. Tedde, E. S. Zaus, J. Fürst, D. Henseler, and P. Lugli, "Active pixel concept combined with organic photodiode for imaging devices," IEEE Electron Dev. Lett. 28, 893-895 (2007).

${ }^{26}$ J. W. Kingsley, S. J. Weston, and D. G. Lidzey, "Stability of X-ray detectors based on organic photovoltaic devices," IEEE J. Sel. Top. Quantum Electron. 16, 1770-1774 (2010).

${ }^{27}$ R. A. Street, X. D. Wu, R. Weisfield, S. Ready, R. Apte, M. Ngyuen, W. B. Jackson, and P. Nylen, "Two-dimensional amorphous silicon image sensor array," J. Non-Cryst. Solid 198-200, 1151-1154 (1996).

${ }^{28}$ J. T. Rahn, F. Lemmi, J. P. Lu, P. Mei, R. B. Apte, R. A. Street, R. Lujan, R. L. Weisfield, and J. A. Heanue, "High resolution x-ray imaging using amorphous silicon flat-panel arrays," IEEE Trans. Nucl. Sci. 46, 457-461 (1999).

${ }^{29}$ M. S. Shur, H. C. Slade, M. D. Jacunski, A. A. Owusu, and T. Ytterdal, "SPICE models for amorphous silicon and polysilicon thin film transistors," J. Elecrochem. Soc. 144, 2833-2839 (1997).

${ }^{30}$ I. A. Cunningham, M. S. Westmore, and A. Fenster, "A spatial-frequency dependent quantum accounting diagram and detective quantum efficiency model of signal to noise propagation in cascaded imaging systems," Med. Phys. 21, 417-427 (1994).

${ }^{31}$ I. A. Cunningham and R. Shaw, "Signal-to-noise optimization of medical imaging systems," J. Opt. Soc. Am. A 16, 621-632 (1999).

${ }^{32}$ J. H. Siewerdsen, L. E. Antonuk, Y. El-Mohri, J. Yorkston, W. Huang, and I. A. Cunningham, "Signal, noise power spectrum, and detective quantum efficiency of indirect-detection flat-panel imagers for diagnostic radiology," Med. Phys. 25, 614-628 (1998).

${ }^{33}$ S. O. Kasap and J. A. Rowlands, "Direct-conversion flat-panel X-ray image sensor for digital radiography," Proc. IEEE 90, 591-604 (2002).

${ }^{34} \mathrm{~W}$. Zhao and J. A. Rowlands, "X-ray imaging using amorphous selenium: Feasibility of a flat panel self-scanned detector for digital radiology," Med. Phys. 22, 1595-1604 (1995).

${ }^{35}$ J. P. Moy, "Image quality of scintillator based X-ray electronic imagers," Proc. SPIE 3336, 14-23 (1998).

${ }^{36}$ Y. El-Mohri, L. E. Antonuk, Q. Zhao, Y. Wang, Y. Lin, H. Du, and A. Sawant, "Performance of a high fill factor, indirect detection prototype flat panel imager for mammography," Med. Phys. 34, 315-327 (2007).

${ }^{37}$ W. Zhao, D. Li, A. Reznik, B. J. M. Lui, D. C. Hunt, J. A. Rowlands, Y. Ohkawa, and K. Tanioka, "Indirect flat-panel detector with avalanche gain: Fundamental feasibility investigation for SHARP-AMFPI (scintillator HARP active matrix flat panel imager)," Med. Phys. 32, 2954-2966 (2005).

${ }^{38}$ R. C. Ronda, Luminescence: From Theory to Applications (Wiley, New York, 2007), pp. 105-132.

${ }^{39}$ T. Hase et al., Phosphor Handbook (CRC, 2000), pp. 539-547.

${ }^{40} \mathrm{~S}$. R. Forrest, "The path to ubiquitous and low-cost organic electronic appliances on plastic," Nature (London) 428, 911-918 (2004).

${ }^{41}$ G. Yu, K. Pakbaz, and A. J. Heeger, "Semiconducting polymer diodes: Large size, low cost photodetectors with excellent visible-ultraviolet sensitivity," Appl. Phys. Lett. 64, 3422-2424 (1994).

${ }^{42}$ T. N. Ng, W. S. Wong, M. L. Chabinyc, S. Sambandan, and R. A. Street, "Flexible image sensor array with bulk heterojunction organic photodiode," Appl. Phys. Lett. 92, 213303 (2008).

${ }^{43}$ B. Park, Y. H. Huh, and M. Kim, "Surfactant additives for improved photovoltaic effect of polymer solar cells," J. Mater. Chem. 20, 10862-10868 (2010).

${ }^{44}$ B. C. Grabmaier, "Crystal scintillator," IEEE Trans. Nucl. Sci. 31, 372-376 (1984).

${ }^{45}$ R. A. Street, S. Nelson, L. Antonuk, and V. Perez Mendez, "Amorphous silicon sensor arrays for radiation imaging," Mater. Res. Soc. Symp. Proc. 192, 441-452 (1992).

${ }^{46}$ Hamamatsu Corp., "FOS (fiber optic plate with scintillator) for digital Xray imaging," Technical Information (Hamamatsu, February 1996).

${ }^{47}$ W. Zhao, G. Ristic, and J. A. Rowlands, "X-ray imaging performance of structured cesium iodide scintillators," Med. Phys. 31, 2594-2605 (2004).

${ }^{48}$ B. Ren, C. Ruth, J. Stein, A. Smith, I. Shaw, and Z. Jing, "Design and performance of the prototype full field breast tomosynthesis system with selenium based flat panel detector," Proc. SPIE 5745, 550-561 (2005).

${ }^{49}$ M. H. Izadi, K. S. Karim, A. Nathan, and J. A. Rowlands, "Low-noise pixel architecture for advanced diagnostic medical x-ray imaging applications," Proc. SPIE 6142, 61420T (2006).

${ }^{50}$ H. Lee, G. Yoo, J.-S. Yoo, and J. Kanicki, "Asymmetric electrical properties of fork a-Si:H thin-film transistor and its applications to flat panel displays," J. Appl. Phys. 105, 124522 (2009).

${ }^{51}$ H. Lee, C.-H. Liu, and J. Kanicki, "Asymmetric electrical properties of half Corbino hydrogenated amorphous silicon thin-film transistor and its applications to flat panel displays," Jpn. J. Appl. Phys. 50, 074203 (2011).

${ }^{52}$ C. Chen, K. Abe, T.-C. Fung, H. Kumomi, and J. Kanicki, "Amorphous In-Ga-Zn-O thin film transistor current-scaling pixel electrode circuit to active-matrix organic light-emitting displays,” Jpn. J. Appl. Phys. 48, 03B025 (2009).

${ }^{53}$ Texas Instruments, "ACF2101 PSpice model," Tools and software (Texas Instruments, October 2000) (available URL: http://www.ti.com/ product/acf2101).

${ }^{54}$ K. Nomura, H. Ohta, A. Takagi, T. Kamiya, M. Hirano, and H. Hosono, "Room-temperature fabrication of transparent flexible thin-film transistors using amorphous oxide semiconductors," Nature (London) 432, 488-492 (2004).

${ }^{55}$ T.-C. Fung, G. Baek, and J. Kanicki, "Low frequency noise in long channel amorphous In-Ga-Zn-O thin film transistors," J. Appl. Phys. 108, 074518 (2010).

${ }^{56}$ T. Arai and T. Sasaoka, "Invited paper: Emergent oxide TFT technologies for next-generation AM-OLED displays," SID Symp. Digest Tech. Pap. 42, 710-713 (2011).

${ }^{57}$ R. Zhang, L. Bie, T.-C. Fung, E. K.-H. Yu, C. Zhao, and J. Kanicki, "High performance amorphous metal-oxide semiconductors thin-film passive and active pixel sensors," in Proceedings of IEEE International Electron Devices Meeting (IEDM) (IEEE, Washington, DC, 2013), pp. 667-670.

${ }^{58}$ A. Mercha, L. Pichon, R. Carin, K. Mourgues, and O. Bonnaud, "Grain boundary trap passivation in polysilicon thin film transistor investigated by low frequency noise," Thin Solid Films 383, 303-306 (2001).

${ }^{59}$ J. Rhayem, D. Rigaud, M. Valenza, N. Szydlo, and H. Lebrun, "1/f noise modeling in long channel amorphous silicon thin film transistors," J. Appl. Phys. 87, 1983-1989 (2000).

${ }^{60} \mathrm{~J}$. N. Boudry and L. E. Antonuk, "Current-noise-power spectra of amorphous silicon thin-film transistors," J. Appl. Phys. 76, 2529-2534 (1994).

${ }^{61}$ S.-W. Lee, and S.-K. Joo, "Low temperature poly-Si thin-film transistor fabrication by metal-induced lateral crystallization," IEEE Electron Dev. Lett. 17, 160-162 (1996).

${ }^{62}$ L. E. Antonuk, Q. Zhao, Y. El-Mohri, H. Du, and Y. Wang, "An investigation of signal performance enhancements achieved through innovative pixel design across several generations of indirect detection, active matrix, flat-panel arrays," Med. Phys. 36, 3322-3339 (2009).

${ }^{63}$ F. R. Libsch and J. Kanicki, "Bias-stress-induced stretched-exponential time dependence of charge injection and trapping in amorphous thin-film transistors," Appl. Phys. Lett. 62, 1286-1288 (1993).

${ }^{64}$ M. H. Izadi and K. S. Karim, "Noise optimization analysis of an active pixel sensor for low-noise real-time fluoroscopy," IET Circuit Dev. Syst. 1, 251-256 (2007).

${ }^{65}$ T. Arai, N. Morosawa, K. Tokunaga, Y. Terai, E. Fukumoto, T. Fujimori, and T. Sasaoka, "Highly reliable oxide semiconductor TFT for AMOLED displays," J. Soc. Inf. Display 19, 205-211 (2011).

${ }^{66}$ J.-S. Park, T.-W. Kim, D. Stryakhilev, J.-S. Lee, S.-G. An, Y.-S. Pyo, D.B. Lee, Y. G. Mo, D. U. Jin, and H. K. Chung, "Flexible full color organic light-emitting diode display on polyimide plastic substrate driven by amorphous indium gallium zinc oxide thin-film transistors," Appl. Phys. Lett. 95, 013503 (2009).

${ }^{67}$ R. A. Lujan and R. A. Street, "Flexible X-ray detector array fabricated with oxide thin-film transistors," IEEE Electron Dev. Lett. 33, 688-690 (2012).

${ }^{68}$ K. Michielsen, K. VanSambrouck, A. Jerebko, and J. Nuyts, "Patchwork reconstruction with resolution modeling for digital breast tomosynthesis," Med. Phys. 40, 031105 (10pp.) (2013). 